\title{
REGULARITY OF SOLUTIONS TO A FRACTIONAL ELLIPTIC PROBLEM WITH MIXED DIRICHLET-NEUMANN BOUNDARY DATA
}

\author{
J. CARMONA, E. COLORADO, T. LEONORI, AND A. ORTEGA
}

\begin{abstract}
In this work we study regularity properties of solutions to fractional elliptic problems with mixed Dirichlet-Neumann boundary data when dealing with the Spectral Fractional Laplacian.
\end{abstract}

\section{INTRODUCTION}

In this paper we study some regularity properties of the solutions to fractional elliptic problems such as

$\left(P^{s}\right) \quad\left\{\begin{aligned}(-\Delta)^{s} u=f & \text { in } \Omega, \\ B(u)=0 & \text { on } \partial \Omega,\end{aligned}\right.$

where $\frac{1}{2}<s<1, f \in L^{p}(\Omega), p>\frac{N}{2 s}$ and $\Omega$ is a bounded domain of $\mathbb{R}^{N}, N \geq 1$. By $B(u)$ we mean the mixed Dirichlet-Neumann boundary condition, i.e.

$$
B(u)=u \chi_{\Sigma_{\mathcal{D}}}+\frac{\partial u}{\partial \nu} \chi_{\Sigma_{\mathcal{N}}}
$$

where $\nu$ is the outwards normal to $\partial \Omega, \chi_{A}$ stands for the characteristic function of the set $A$ and $\Omega$ satisfy

$$
\left\{\begin{array}{c}
\Omega \subset \mathbb{R}^{N} \text { is a bounded Lipschitz domain } \\
\Sigma_{\mathcal{D}} \text { and } \Sigma_{\mathcal{N}} \text { are smooth }(N-1) \text {-dimensional submanifolds of } \partial \Omega \\
\Sigma_{\mathcal{D}} \text { is a closed manifold of positive }(N-1) \text {-dimensional Lebesgue measure, } \\
\left|\Sigma_{\mathcal{D}}\right|=\alpha \in(0,|\partial \Omega|) \\
\Sigma_{\mathcal{D}} \cap \Sigma_{\mathcal{N}}=\emptyset, \Sigma_{\mathcal{D}} \cup \Sigma_{\mathcal{N}}=\partial \Omega \text { and } \Sigma_{\mathcal{D}} \cap \bar{\Sigma}_{\mathcal{N}}=\Gamma \\
\Gamma \text { is a smooth }(N-2) \text {-dimensional submanifold of } \partial \Omega
\end{array}\right.
$$

The main result we prove here is the following.

Theorem 1.1. Assume that $\Omega$ satisfies hypotheses $(\mathfrak{B})$ and let $u$ be the solution to problem $\left(P^{s}\right.$ with $\frac{1}{2}<s<1, f \in L^{p}(\Omega), p>\frac{N}{2 s}$. Then $u \in \mathcal{C}^{\gamma}(\bar{\Omega})$ for some $0<\gamma<\frac{1}{2}$. Moreover, there exists a constant $\mathscr{H}=\mathscr{H}\left(N, s, f, p,\left|\Sigma_{\mathcal{D}}\right|\right)>0$ such that

$$
|u(x)-u(y)| \leq \mathscr{H}|x-y|^{\gamma}, \forall x, y \in \bar{\Omega} .
$$

To prove Theorem 1.1 we follow some of the ideas in [8, 10. Using the De Giorgi truncation method, Stampacchia (see [10]) established the regularity of solutions to the mixed boundary problem involving the classical Laplace operator. Due to the nonlocal nature of problem $\left(\overline{P^{s}}\right)$, some difficulties arise when trying to apply this truncation method to solutions to $\left(P^{s}\right.$. Based on the ideas of [2, 3, 1, at this point we will make full use of the local realization of the fractional operator $(-\Delta)^{s}$ in terms of certain auxiliary degenerate elliptic problem. We use the results of [7] to adapt the procedures of

Date: March 27, 2019.

2010 Mathematics Subject Classification. 35R11, 35B655.

Key words and phrases. Fractional Laplacian, Mixed Boundary Conditions, Regularity.

E. Colorado and A. Ortega are partially supported by the Ministry of Economy and Competitiveness of Spain and FEDER under Research Project MTM2016-80618-P. J. Carmona is partially supported by Ministerio de Economía y Competitividad (MINECO-FEDER), Spain under grant MTM2015-68210-P and Junta de Andalucía FQM-194. 
[10] to the case of degenerate elliptic equations with weights in the Muckenhoupt class $A_{2}$ (see [7] for the precise definition as well as some useful properties of those weights).

In addition to Theorem 1.1. following some ideas in [6, in the last part of the work we study the behaviour of the problem $\left(\overline{P^{s}}\right.$ ) when we move the boundary condition in a regular way as follows. Given $I_{\varepsilon}=[\varepsilon,|\partial \Omega|]$ for some $\varepsilon>0$, let us consider the family of closed sets $\left\{\Sigma_{\mathcal{D}}(\alpha)\right\}_{\alpha \in I_{\varepsilon}}$, satisfying

$\left(B_{1}\right) \Sigma_{\mathcal{D}}(\alpha)$ has a finite number of connected components.

$\left(B_{2}\right) \Sigma_{\mathcal{D}}\left(\alpha_{1}\right) \subset \Sigma_{\mathcal{D}}\left(\alpha_{2}\right)$ if $\alpha_{1}<\alpha_{2}$.

$\left(B_{3}\right)\left|\Sigma_{\mathcal{D}}\left(\alpha_{1}\right)\right|=\alpha_{1} \in I_{\varepsilon}$.

We denote by $\Sigma_{\mathcal{N}}(\alpha)=\partial \Omega \backslash \Sigma_{\mathcal{D}}(\alpha)$ and $\Gamma(\alpha)=\Sigma_{\mathcal{D}}(\alpha) \cap \bar{\Sigma}_{\mathcal{N}}(\alpha)$. For a family of this type we consider the corresponding family of mixed boundary value problems

$$
\left\{\begin{aligned}
(-\Delta)^{s} u=f & & \text { in } \Omega \subset \mathbb{R}^{n}, \\
B_{\alpha}(u)=0 & & \text { on } \partial \Omega,
\end{aligned}\right.
$$

where $B_{\alpha}(u)$ is the boundary condition associated to the parameter $\alpha$ in the previous hypotheses and the boundary manifolds $\Sigma_{\mathcal{D}}(\alpha)$ and $\Sigma_{\mathcal{N}}(\alpha)$ satisfy the corresponding hypotheses $\left(\mathfrak{B}_{\alpha}\right)$. In this scenario we prove the following result.

Theorem 1.2. Given $\Omega$ a smooth bounded domain such that the family $\left\{\Sigma_{\mathcal{D}}(\alpha)\right\}_{\alpha \in I_{\varepsilon}}$ satisfies the hypotheses $\left(\mathfrak{B}_{\alpha}\right)$ and $\left(B_{1}\right)-\left(B_{3}\right)$, let $u_{\alpha}$ be the solution to $\left(\overline{P_{\alpha}^{s}}\right)$ with $\frac{1}{2}<s<1, f \in L^{p}(\Omega)$ and $p>\frac{N}{2 s}$. Then, there exist two constants $0<\gamma<\frac{1}{2}$ and $\mathscr{H}_{\varepsilon}>0$ both independent from $\alpha \in[\varepsilon,|\partial \Omega|]$ such that

$$
\left\|u_{\alpha}\right\|_{\mathcal{C}^{\gamma}(\bar{\Omega})} \leq \mathscr{H}_{\varepsilon}
$$

As we will see in the proof of Theorem 1.2, when one takes $\alpha \rightarrow 0^{+}$the control of the Hölder norm of such a family is lost. Hence, it is necessary bound from below the measure of the family $\left\{\Sigma_{\mathcal{D}}(\alpha)\right\}_{\alpha \in I_{\varepsilon}}$, in order to guarantee the control on the Hölder norm for the family $\left\{u_{\alpha}\right\}_{\alpha \in I_{\varepsilon}}$.

Let us stress that problem related to the spectral fractional Laplacian with mixed boundary conditions are news and, to our knowledge, have been treated only in 4,5 .

\section{FunCtional SETting AND PRELIMINARIES}

As far as the fractional Laplace operator is concerned, we recall its definition given through the spectral decomposition. Let $\left(\varphi_{i}, \lambda_{i}\right)$ be the eigenfunctions (normalized with respect to the $L^{2}(\Omega)$ norm) and the eigenvalues of $(-\Delta)$ equipped with homogeneous mixed Dirichlet-Neumann boundary data. Then, $\left(\varphi_{i}, \lambda_{i}^{s}\right)$ are the eigenfunctions and eigenvalues of the fractional operator $(-\Delta)^{s}$, where given $u_{i}(x)=\sum_{j \geq 1}\left\langle u_{i}, \varphi_{j}\right\rangle \varphi_{j}, i=1,2$

$$
\left\langle(-\Delta)^{s} u_{1}, u_{2}\right\rangle=\sum_{j \geq 1} \lambda_{j}^{s}\left\langle u_{1}, \varphi_{j}\right\rangle\left\langle u_{2}, \varphi_{j}\right\rangle
$$

i.e., the action of the fractional operator on a smooth function $u_{1}$ is given by

$$
(-\Delta)^{s} u_{1}=\sum_{j \geq 1} \lambda_{j}^{s}\left\langle u_{1}, \varphi_{j}\right\rangle \varphi_{j}
$$

As a consequence, the fractional Laplace operator $(-\Delta)^{s}$ is well defined through its spectral decomposition in the following space of functions that vanish on $\Sigma_{\mathcal{D}}$,

$$
H_{\Sigma_{\mathcal{D}}}^{s}(\Omega)=\left\{u=\sum_{j \geq 1} a_{j} \varphi_{j} \in L^{2}(\Omega):\|u\|_{H_{\Sigma_{\mathcal{D}}^{s}}(\Omega)}^{2}=\sum_{j \geq 1} a_{j}^{2} \lambda_{j}^{s}<\infty\right\} .
$$

Observe that since $u \in H_{\Sigma_{\mathcal{D}}}^{s}(\Omega)$, it follows that

$$
\|u\|_{H_{\Sigma_{\mathcal{D}}}^{s}(\Omega)}=\left\|(-\Delta)^{\frac{s}{2}} u\right\|_{L^{2}(\Omega)} .
$$


As it is proved in [9, Theorem 11.1], if $0<s \leq \frac{1}{2}$ then $H_{0}^{s}(\Omega)=H^{s}(\Omega)$ and, therefore, also $H_{\Sigma_{\mathcal{D}}}^{s}(\Omega)=H^{s}(\Omega)$, while for $\frac{1}{2}<s<1, H_{0}^{s}(\Omega) \subsetneq H^{s}(\Omega)$. Hence, the range $\frac{1}{2}<s<1$ guarantees that $H_{\Sigma_{\mathcal{D}}}^{s}(\Omega) \subsetneq H^{s}(\Omega)$, provides us the correct functional space to study the mixed boundary problem $\left(\overrightarrow{P^{s}}\right)$.

This definition of the fractional powers of the Laplace operator allows us to integrate by parts in the appropriate spaces, so that a natural definition of weak solution to problem $\left(P_{s}\right)$ is the following.

Definition 2.1. We say that $u \in H_{\Sigma_{\mathcal{D}}}^{s}(\Omega)$ is a solution to $\left(\underline{P^{s}}\right)$ if

$$
\int_{\Omega}(-\Delta)^{s / 2} u(-\Delta)^{s / 2} \psi d x=\int_{\Omega} f \psi d x, \text { for any } \psi \in H_{\Sigma_{\mathcal{D}}}^{s}(\Omega) \text {. }
$$

Due to the nonlocal nature of the fractional operator $(-\Delta)^{s}$ some difficulties arise when one tries to obtain an explicit expression of the action of the fractional Laplacian on a given function. In order to overcome this difficuly, we use the ideas by Caffarelli and Silvestre (see [2]) together with those of [1, 3. to give an equivalent definition of the operator $(-\Delta)^{s}$ by means of an auxiliary problem that we introduce next.

Given any domain $\Omega \subset \mathbb{R}^{N}$, we set the cylinder $\mathscr{C}_{\Omega}=\Omega \times(0, \infty) \subset \mathbb{R}_{+}^{N+1}$. We denote by $(x, y)$ those points that belong to $\mathscr{C}_{\Omega}$ and by $\partial_{L} \mathscr{C}_{\Omega}=\partial \Omega \times[0, \infty)$ the lateral boundary of the cylinder. Let us also denote by $\Sigma_{\mathcal{D}}^{*}=\Sigma_{\mathcal{D}} \times[0, \infty)$ and $\Sigma_{\mathcal{N}}^{*}=\Sigma_{\mathcal{N}} \times[0, \infty)$ as well as $\Gamma^{*}=\Gamma \times[0, \infty)$. It is clear that, by construction,

$$
\Sigma_{\mathcal{D}}^{*} \cap \Sigma_{\mathcal{N}}^{*}=\emptyset, \quad \Sigma_{\mathcal{D}}^{*} \cup \Sigma_{\mathcal{N}}^{*}=\partial_{L} \mathscr{C}_{\Omega} \quad \text { and } \quad \Sigma_{\mathcal{D}}^{*} \cap \overline{\Sigma_{\mathcal{N}}^{*}}=\Gamma^{*}
$$

Given a function $u \in H_{\Sigma_{\mathcal{D}}}^{s}(\Omega)$ we define its $s$-harmonic extension function, denoted by $U(x, y)=$ $E_{s}[u(x)]$, as the solution to the problem

$$
\left\{\begin{aligned}
-\operatorname{div}\left(y^{1-2 s} \nabla U(x, y)\right) & =0 & & \text { in } \mathscr{C}_{\Omega}, \\
B(U(x, y)) & =0 & & \text { on } \partial_{L} \mathscr{C}_{\Omega}, \\
U(x, 0) & =u(x) & & \text { on } \Omega \times\{y=0\} .
\end{aligned}\right.
$$

where

$$
B(U)=U \chi_{\Sigma_{\mathcal{D}}^{*}}+\frac{\partial U}{\partial \nu} \chi_{\Sigma_{\mathcal{N}}^{*}}
$$

being $\nu$, with an abuse of notation 1 , the outwards normal to $\partial_{L} \mathscr{C}_{\Omega}$. Following the well known result by Caffarelli and Silvestre (see [2]), $U$ is related to the fractional Laplacian of the original function through the formula

$$
\frac{\partial U}{\partial \nu^{s}}:=-\kappa_{s} \lim _{y \rightarrow 0^{+}} y^{1-2 s} \frac{\partial U}{\partial y}=(-\Delta)^{s} u(x),
$$

where $\kappa_{s}$ is a suitable positive constant (see [1] for its exact value). The extension function belongs to the space

where we define

$$
\mathcal{X}_{\Sigma_{\mathcal{D}}}^{s}\left(\mathscr{C}_{\Omega}\right):=\overline{\mathcal{C}_{0}^{\infty}\left(\left(\Omega \cup \Sigma_{\mathcal{N}}\right) \times[0, \infty)\right)} \|^{\|\cdot\|_{\mathcal{X}_{\mathcal{D}_{\mathcal{D}}}^{s}}\left(\mathscr{C}_{\Omega}\right)},
$$

$$
\|\cdot\|_{\mathcal{X}_{\Sigma_{\mathcal{D}}}^{s}\left(\mathscr{C}_{\Omega}\right)}^{2}:=\kappa_{s} \int_{\mathscr{C}_{\Omega}} y^{1-2 s}|\nabla(\cdot)|^{2} d x d y .
$$

Note that $\mathcal{X}_{\Sigma_{\mathcal{D}}}^{s}\left(\mathscr{C}_{\Omega}\right)$ is a Hilbert space equipped with the norm $\|\cdot\|_{\mathcal{X}_{\Sigma_{\mathcal{D}}}^{s}\left(\mathscr{C}_{\Omega}\right)}$ which is induced by the scalar product

$$
\langle U, V\rangle_{\mathcal{X}_{\Sigma_{\mathcal{D}}}^{s}\left(\mathscr{C}_{\Omega}\right)}=\kappa_{s} \int_{\mathscr{C}_{\Omega}} y^{1-2 s}\langle\nabla U, \nabla V\rangle d x d y
$$

Moreover, the following inclusions are satisfied,

$$
\mathcal{X}_{0}^{s}\left(\mathscr{C}_{\Omega}\right) \subset \mathcal{X}_{\Sigma_{\mathcal{D}}}^{s}\left(\mathscr{C}_{\Omega}\right) \subsetneq \mathcal{X}^{s}\left(\mathscr{C}_{\Omega}\right)
$$

being $\mathcal{X}_{0}^{s}\left(\mathscr{C}_{\Omega}\right)$ the space of functions that belongs to $\mathcal{X}^{s}\left(\mathscr{C}_{\Omega}\right) \equiv H^{1}\left(\mathscr{C}_{\Omega}, y^{1-2 s} d x d y\right)$ and vanish on the lateral boundary of $\mathscr{C}_{\Omega}$.

\footnotetext{
${ }^{1}$ Let $\nu$ be the outwards normal to $\partial \Omega$ and $\nu_{(x, y)}$ the outwards normal to $\mathscr{C}_{\Omega}$ then, by construction, $\nu_{(x, y)}=(\nu, 0)$, $y>0$.
} 
Using the above arguments we can reformulate the problem $\left(\underline{P^{s}}\right)$ in terms of the extension problem as follows:

$$
\left\{\begin{aligned}
-\operatorname{div}\left(y^{1-2 s} \nabla U\right) & =0 & & \text { in } \mathscr{C}_{\Omega}, \\
B(U) & =0 & & \text { on } \partial_{L} \mathscr{C}_{\Omega}, \\
\frac{\partial U}{\partial \nu^{s}} & =f & & \text { on } \Omega \times\{y=0\} .
\end{aligned}\right.
$$

Next, we specify

Definition 2.2. An energy solution to problem $\left[P_{s}^{*}\right.$ is a function $U \in \mathcal{X}_{\Sigma_{\mathcal{D}}}^{s}\left(\mathscr{C}_{\Omega}\right)$ such that

$$
\kappa_{s} \int_{\mathscr{C}_{\Omega}} y^{1-2 s}\langle\nabla U, \nabla \varphi\rangle d x d y=\int_{\Omega} f(x) \varphi(x, 0) d x, \quad \forall \varphi \in \mathcal{X}_{\Sigma_{\mathcal{D}}}^{s}\left(\mathscr{C}_{\Omega}\right) .
$$

If $U \in \mathcal{X}_{\Sigma_{\mathcal{D}}}^{s}\left(\mathscr{C}_{\Omega}\right)$ is the solution to problem $\left(P_{s}^{*}\right)$ we can associate the function $u(x)=\operatorname{Tr}[U(x, y)]=$ $U(x, 0)$, that belongs to $H_{\Sigma_{\mathcal{D}}}^{s}(\Omega)$, and solves problem $\left(\overrightarrow{P^{s}}\right)$. Moreover, also the vice versa is true: given a solution $u \in H_{\Sigma_{\mathcal{D}}}^{s}(\Omega)$ we can define its $s$-harmonic extension $U \in \mathcal{X}_{\Sigma_{\mathcal{D}}}^{s}\left(\mathscr{C}_{\Omega}\right)$, as the solution to $\left(P_{s}^{*}\right\rangle$. Thus, both formulations are equivalent and the Extension operator

$$
E_{s}: H_{\Sigma_{\mathcal{D}}}^{s}(\Omega) \rightarrow \mathcal{X}_{\Sigma_{\mathcal{D}}}^{s}\left(\mathscr{C}_{\Omega}\right)
$$

allows us to switch between both of them.

Accordingly to 2, 1], due to the choice of the constant $\kappa_{s}$, the extension operator $E_{s}$ is an isometry, i.e.

$$
\left\|E_{s}[\varphi](x, y)\right\|_{\mathcal{X}_{\Sigma_{\mathcal{D}}}^{s}\left(\mathscr{C}_{\Omega}\right)}=\|\varphi(x)\|_{H_{\Sigma_{\mathcal{D}}}^{s}(\Omega)} \text {, for all } \varphi \in H_{\Sigma_{\mathcal{D}}}^{s}(\Omega) .
$$

Let us also recall the trace inequality, that is a useful tool we exploit in many proofs in this paper (see [1]):

there exists $C=C(N, s, r,|\Omega|)$ such that $\forall z \in \mathcal{X}_{0}^{s}\left(\mathscr{C}_{\Omega}\right)$

$$
C\left(\int_{\Omega}|z(x, 0)|^{r} d x\right)^{\frac{2}{r}} \leq \int_{\mathscr{C}_{\Omega}} y^{1-2 s}|\nabla z(x, y)|^{2} d x d y
$$

with $1 \leq r \leq 2_{s}^{*}, N>2 s$, with $2_{s}^{*}=\frac{2 N}{N-2 s}$.

Observe that such inequality turns out to be, in fact, equivalent to the fractional Sobolev inequality:

$$
C\left(\int_{\Omega}|v|^{r} d x\right)^{\frac{2}{r}} \leq \int_{\Omega}\left|(-\Delta)^{\frac{s}{2}} v\right|^{2} d x, \quad \forall v \in H_{0}^{s}(\Omega), 1 \leq r \leq 2_{s}^{*}, N>2 s .
$$

When mixed boundary conditions are considered, the situation is quite similar since the Dirichlet condition is imposed on a set $\Sigma_{\mathcal{D}} \subset \partial \Omega$ such that $\left|\Sigma_{\mathcal{D}}\right|=\alpha>0$. Hence, thanks to (2.1), there exists a positive constant $C_{\mathcal{D}}=C_{\mathcal{D}}\left(N, s,\left|\Sigma_{\mathcal{D}}\right|\right)$ such that

$$
0<\inf _{\substack{u \in H_{\Sigma_{\mathcal{D}}}^{s}(\Omega) \\ u \neq 0}} \frac{\|u\|_{H_{\Sigma_{\mathcal{D}}}^{s}(\Omega)}^{2}}{\|u\|_{L^{2 *}(\Omega)}^{2}}:=C_{\mathcal{D}}<\inf _{\substack{u \in H_{0}^{s}(\Omega) \\ u \neq 0}} \frac{\|u\|_{H_{0}^{s}(\Omega)}^{2}}{\|u\|_{L^{2 *}(\Omega)}^{2}} .
$$

Remark 2.1. It is worth to observe (see [5], 4]) that $C_{\mathcal{D}}\left(N, s,\left|\Sigma_{\mathcal{D}}\right|\right) \leq 2^{-\frac{2 s}{N}} C\left(N, s, 2_{s}^{*}\right)$. Moreover, having in mind the spectral definition of the fractional operator and by Hölder inequality, it follows that $C_{\mathcal{D}} \leq|\Omega|^{\frac{2 s}{N}} \lambda_{1}^{s}(\alpha)$, with $\lambda_{1}(\alpha)$ the first eigenvalue of the Laplace operator with mixed boundary conditions on the sets $\Sigma_{\mathcal{D}}=\Sigma_{\mathcal{D}}(\alpha)$ and $\Sigma_{\mathcal{N}}=\Sigma_{\mathcal{N}}(\alpha)$. Since $\lambda_{1}(\alpha) \rightarrow 0$ as $\alpha \rightarrow 0^{+}$, see [6, Lemma 4.3], we conclude that $C_{\mathcal{D}} \rightarrow 0$ as $\alpha \rightarrow 0^{+}$.

Gathering together (2.3) and (2.4), we obtain,

$$
C_{\mathcal{D}}\left(\int_{\Omega}|\varphi(x, 0)|^{2_{s}^{*}} d x\right)^{\frac{2}{2_{s}^{*}}} \leq\|\varphi(x, 0)\|_{H_{\Sigma_{\mathcal{D}}}^{s}(\Omega)}^{2}=\left\|E_{s}[\varphi(x, 0)]\right\|_{\mathcal{X}_{\Sigma_{\mathcal{D}}^{s}}\left(\mathscr{C}_{\Omega}\right)}^{2}
$$

With this Sobolev-type inequality in hand we can prove a trace inequality adapted to the mixed boundary data framework. 
Lemma 2.1. There exists a constant $C_{\mathcal{D}}=C_{\mathcal{D}}\left(N, s,\left|\Sigma_{\mathcal{D}}\right|\right)>0$ such that,

$$
\left.C_{\mathcal{D}}\left(\int_{\Omega}|\varphi(x, 0)|^{2_{s}^{*}}\right) d x\right)^{\frac{2}{2_{s}^{*}}} \leq \int_{\mathscr{C}_{\Omega}} y^{1-2 s}|\nabla \varphi|^{2} d x d y, \quad \forall \varphi \in \mathcal{X}_{\Sigma_{\mathcal{D}}}^{s}\left(\mathscr{C}_{\Omega}\right)
$$

Proof. Thanks to (2.5), it is enough to prove that $\left\|E_{s}[\varphi(\cdot, 0)]\right\|_{\mathcal{X}_{\Sigma_{\mathcal{D}}}^{s}\left(\mathscr{C}_{\Omega}\right)} \leq\|\varphi\|_{\mathcal{X}_{\Sigma_{\mathcal{D}}}^{s}\left(\mathscr{C}_{\Omega}\right)}$. This inequality is satisfied since, arguing as in [1, we find

$$
\begin{aligned}
\|\varphi\|_{\mathcal{X}_{\Sigma_{\mathcal{D}}}^{s}\left(\mathscr{C}_{\Omega}\right)}^{2} & =\kappa_{s} \int_{\mathscr{C}_{\Omega}} y^{1-2 s}|\nabla \varphi|^{2} d x d y \\
& =\kappa_{s} \int_{\mathscr{C}_{\Omega}} y^{1-2 s}\left|\nabla\left(E_{s}[\varphi(x, 0)]+\varphi(x, y)-E_{s}[\varphi(x, 0)]\right)\right|^{2} d x d y \\
& =\left\|E_{s}[\varphi(x, 0)]\right\|_{\mathcal{X}_{\Sigma_{\mathcal{D}}}^{s}\left(\mathscr{C}_{\Omega}\right)}^{2}+\|\varphi(x, y)-E(\varphi(x, 0))\|_{\mathcal{X}_{\Sigma_{\mathcal{D}}}^{s}\left(\mathscr{C}_{\Omega}\right)}^{2} \\
& +2 \kappa_{s} \int_{\mathscr{C}_{\Omega}} y^{1-2 s}\left\langle\nabla E_{s}[\varphi(x, 0)], \nabla\left(\varphi(x, y)-E_{s}[\varphi(x, 0)]\right)\right\rangle d x d y \\
& =\left\|E_{s}[\varphi(x, 0)]\right\|_{\mathcal{X}_{\Sigma_{\mathcal{D}}}^{s}\left(\mathscr{C}_{\Omega}\right)}^{2}+\left\|\varphi(x, y)-E_{s}[\varphi(x, 0)]\right\|_{\mathcal{X}_{\Sigma_{\mathcal{D}}}^{s}}^{2}\left(\mathscr{C}_{\Omega}\right) \\
& +2 \int_{\Omega}(-\Delta)^{s}(\varphi(x, 0))(\varphi(x, 0)-\varphi(x, 0)) d x \\
& =\left\|E_{s}[\varphi(x, 0)]\right\|_{\mathcal{X}_{\Sigma_{\mathcal{D}}}^{s}\left(\mathscr{C}_{\Omega}\right)}^{2}+\left\|\varphi(x, y)-E_{s}[\varphi(x, 0)]\right\|_{\mathcal{X}_{\Sigma_{\mathcal{D}}}^{s}}^{2} \mathscr{C}_{\Omega} .
\end{aligned}
$$

\section{HöLder REgularity}

The principal result we prove in this Section is Theorem 1.1 which deals with the Hölder regularity of the solution to problem $\left(\overline{P^{s}}\right)$. First we introduce the notation that we will follow along this Section.

Notation. Given an open bounded set $\Omega, x \in \bar{\Omega} \subset \mathbb{R}^{N}$ and $X \in \overline{\mathscr{C}}_{\Omega} \subset \mathbb{R}_{+}^{N+1}$, we define

$-\Omega(x, \rho)=\Omega \cap B_{\rho}(x)$

- $\mathscr{C}_{\Omega}(X, \rho)=\mathscr{C}_{\Omega} \cap B_{\rho}(X)$,

Given $u(x) \in H_{\Sigma_{\mathcal{D}}}^{s}(\Omega)$ and $U(X) \in \mathcal{X}_{\Sigma_{\mathcal{D}}}^{s}\left(\mathscr{C}_{\Omega}\right)$, let us also define

- $A_{+}(k)=\{x \in \Omega: u(x)>k\}$,

- $A_{+}^{*}(k)=\left\{X \in \mathscr{C}_{\Omega}: U(X)>k\right\}$,

- $A_{+}(k, \rho)=A_{+}(k) \cap \Omega(x, \rho)$

- $A_{+}^{*}(k, \rho)=A_{+}^{*}(k) \cap \mathscr{C}_{\Omega}(X, \rho)$,

$-\{\cdot\}^{k}=\min (\cdot, k)$.

$-\{\cdot\}_{k}=\max (\cdot, k)$.

In a similar way we may define the sets $A_{-}(k), A_{-}^{*}(k), A_{-}(k, \rho)$ and $A_{-}^{*}(k, \rho)$ replacing $>$ with $<$ in the latter definitions. We denote by

- $|A|_{\omega}$ the measure induced by a weight $\omega$ of the set $A$.

- $|A|_{y^{1-2 s}}$ the measure induced by the weight $y^{1-2 s}$ of the set $A$.

- $|A|$ the usual Lebesgue measure of the set $A$.

On the regularity of $\Omega$. Let us recall that $\Omega$ is assumed, in all the paper, to be Lipschitz and consequently also $\mathcal{C}_{\Omega}$ turns out to have the same regularity. In particular, among others, we use the following properties. There exists $\zeta \in(0,1)$ such that for any $z \in \bar{\Omega}$ and any $\rho>0$

$$
\left|\mathscr{C}_{\Omega}(Z, \rho)\right| \geq \zeta\left|B_{\rho}(Z)\right| .
$$

Moreover also the weighted counterpart is true, i.e. there exists $\zeta_{s} \in(0,1)$ such that for any $z \in \bar{\Omega}$ and any $\rho>0$

$$
\left|\mathscr{C}_{\Omega}(Z, \rho)\right|_{y^{1-2 s}} \geq \zeta_{s}\left|B_{\rho}(Z)\right|_{y^{1-2 s}} .
$$


Consequently $\exists \lambda>0$ such that

$$
\left|A_{+}^{*}(k, r)\right|_{y^{1-2 s}} \leq \lambda\left|\mathscr{C}_{\Omega(z, R)}(Z, r)\right|_{y^{1-2 s}} .
$$

It is worth to observe that all the results we prove in this paper might be proved for a larger class of open sets $\Omega$. Indeed following [10, this kind of results is true for the so called $\frac{1}{2}$-admissible domains. Here we decided to not deal with such domains for brevity and in order to not make the proofs much heavier.

Now we are ready to start with the statement and the proofs of several technical results.

Let $z \in \bar{\Omega}$ and $R>0$ and let $u$ be a solution to problem $\left(\underline{P^{s}}\right)$ : we write $u(x)=v(x)+w(x)$ for every $x \in \Omega(z, R)$, where the function $v(x)$ satisfies

$$
\left\{\begin{aligned}
(-\Delta)^{s} v=f & \text { in } \Omega(z, R), \\
v=0 & \text { on } \widetilde{\Sigma}_{\mathcal{D}, R}:=\partial \Omega(z, R) \backslash \Sigma_{\mathcal{N}} \\
\frac{\partial v}{\partial \nu}=0 & \text { on } \widetilde{\Sigma}_{\mathcal{N}, R}:=\partial \Omega(z, R) \cap \Sigma_{\mathcal{N}}
\end{aligned}\right.
$$

and the function $w(x)$ is such that,

$$
\left\{\begin{array}{rlrl}
(-\Delta)^{s} w=0 & & \text { in } \Omega(z, R), \\
w & =0 & & \text { on } \Sigma_{\mathcal{D}, R}:=\Sigma_{\mathcal{D}} \cap B_{R}(z), \\
\frac{\partial w}{\partial \nu}=0 & & \text { on } \Sigma_{\mathcal{N}, R}:=\Sigma_{\mathcal{N}} \cap B_{R}(z),
\end{array}\right.
$$

Using the extension technique we can write $v(x)=V(x, 0)$ with $V(x, y)$ solves the extended problem

$$
\left\{\begin{array}{rlrl}
-\operatorname{div}\left(y^{1-2 s} \nabla V\right)=0 & & \text { in } \mathscr{C}_{\Omega(z, R)}, \\
B(V)=0 & & \text { on } \partial_{L} \mathscr{C}_{\Omega(z, R)}, \\
\frac{\partial V}{\partial \nu^{s}} & =f & & \text { on } \Omega(z, R) \times\{y=0\},
\end{array}\right.
$$

where $B(V)=V \chi_{\widetilde{\Sigma}_{\mathcal{D}, R}^{*}}+\frac{\partial V}{\partial \nu} \chi_{\widetilde{\Sigma}_{\mathcal{D}, R}^{*}}$, with $\widetilde{\Sigma}_{\mathcal{D}, R}^{*}=\widetilde{\Sigma}_{\mathcal{D}, R} \times[0, \infty)$ and $\widetilde{\Sigma}_{\mathcal{N}, R}^{*}=\widetilde{\Sigma}_{\mathcal{N}, R} \times[0, \infty)$.

In the same way, we write $w(x)=W(x, 0)$, with $W(x, y)$ satisfying the extended problem

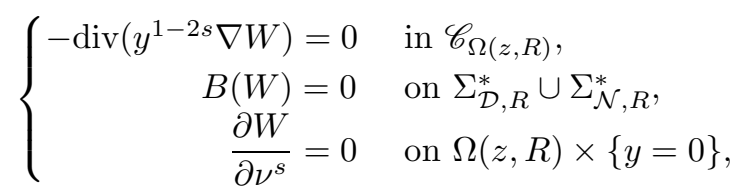

where $B(V)=V \chi_{\Sigma_{\mathcal{D}, R}^{*}}+\frac{\partial V}{\partial \nu} \chi_{\Sigma_{\mathcal{D}, R}^{*}}$, with $\Sigma_{\mathcal{D}, R}^{*}=\Sigma_{\mathcal{D}, R} \times[0, \infty)$ and $\Sigma_{\mathcal{N}, R}^{*}=\Sigma_{\mathcal{N}, R} \times[0, \infty)$.

Let us observe that we have the following situations:

(i) If $z \in \Omega$, there exists $R>0$ such that $\widetilde{\Sigma}_{\mathcal{D}, R}=\partial \Omega(z, R)$ and $\Sigma_{\mathcal{D}, R}=\Sigma_{\mathcal{N}, R}=\emptyset$. Then, $v \in H_{0}^{s}(\Omega(z, R))$ and it is solution to a Dirichlet problem. Moreover, $w$ is an $s$-harmonic function, i.e. its extension $W=E_{s}[w] \in \mathcal{X}^{s}\left(\mathscr{C}_{\Omega(z, R)}\right)$ and it satisfies

$$
\int_{\mathscr{C}_{\Omega(z, R)}} y^{1-2 s}\langle\nabla W, \nabla \Phi\rangle d x d y=0, \quad \forall \Phi \in \mathcal{X}_{0}^{s}\left(\mathscr{C}_{\Omega(z, R)}\right) .
$$

(ii) If $z \in \Sigma_{\mathcal{D}} \backslash \Gamma$, there exists $R>0$ such that $\widetilde{\Sigma}_{\mathcal{D}, R}=\partial \Omega(z, R)$ and $\Sigma_{\mathcal{N}, R}=\emptyset$, then, $v \in$ $H_{0}^{s}(\Omega(z, R))$ and it is a solution to a Dirichlet problem while $W \in \mathcal{X}_{\Sigma_{\mathcal{D}, R}}^{s}\left(\mathscr{C}_{\Omega(z, R)}\right)$ and, also in this case, it satisfies (3.8).

(iii) If $z \in \Sigma_{\mathcal{N}}$, there exists $R>0$ such that $\Sigma_{\mathcal{D}, R}=\emptyset$. Then, the function $v \in H_{\widetilde{\Sigma}_{\mathcal{D}, R}}^{s}(\Omega(z, R))$ and it is a solution to the mixed problem (3.4); moreover $W$ belongs to $\mathcal{X}^{s}\left(\mathscr{C}_{\Omega(z, R)}\right)$ and (3.8) holds $\forall \Phi \in \mathcal{X}^{s}\left(\mathscr{C}_{\Omega(z, R)}\right)$ vanishing on $\partial_{L} \mathscr{C}_{\Omega(z, R)} \backslash \Sigma_{\mathcal{N}, R}^{*}$. 
(iv) Finally, if $z \in \Gamma$, the sets $\widetilde{\Sigma}_{\mathcal{D}, R}, \widetilde{\Sigma}_{\mathcal{N}, R}, \Sigma_{\mathcal{D}, R}$ and $\Sigma_{\mathcal{D}, R}$ are nonempty for all $R>0$. Then, the function $v \in H_{\widetilde{\Sigma}_{\mathcal{D}, R}}^{s}(\Omega(z, R))$ and it is a solution to the mixed problem (3.4); as far as $w$ is concerned, $W \in \mathcal{X}_{\Sigma_{\mathcal{D}, R}^{s}}\left(\mathscr{C}_{\Omega(z, R)}\right)$ and if fulfills (3.8) holds for any $\Phi \in \mathcal{X}^{s}\left(\mathscr{C}_{\Omega(z, R)}\right)$ vanishing on $\partial_{L} \mathscr{C}_{\Omega(z, R)} \backslash \Sigma_{\mathcal{N}, R}^{*}$.

We also define the following sets that will be useful in the sequel:

- $\mathscr{C}_{\Omega(z, R)}^{\circ}=\overline{\mathscr{C}}_{\Omega(z, R)} \backslash\left\{(x, y) \in \mathscr{C}_{\Omega(z, R)}: x \in \partial B_{R}(z)\right\}$,

- $\partial_{0} \mathscr{C}_{\Omega(z, R)}=\partial_{L} \mathscr{C}_{\Omega(z, R)} \backslash \Sigma_{\mathcal{N}, R}^{*}$.

- $\partial_{B} \mathscr{C}_{\Omega(z, R)}=\partial_{L} \mathscr{C}_{\Omega(z, R)} \backslash\left(\Sigma_{\mathcal{D}, R}^{*} \cup \Sigma_{\mathcal{N}, R}^{*}\right)$.

We continue by stating the definitions and results needed in what follows. The first definition is based on [10, Definition 2.1].

Definition 3.1. Given any $z_{0} \in \bar{\Omega}$ and $Z \in \mathscr{C}_{\Omega\left(z_{0}, R\right)}^{\circ}$, let $\mathcal{K}^{+}(Z)\left(\right.$ resp. $\left.\mathcal{K}^{-}(Z)\right)$ be the set of values $k \in \mathbb{R}$ such that there exists a number $\widetilde{\rho}(Z)>0$ satisfying $\{U\}^{k} \eta \in \mathcal{X}_{\partial_{0} \mathscr{C}_{\Omega\left(z_{0}, R\right)}}\left(\mathscr{C}_{\Omega\left(z_{0}, R\right)}\right)$ (resp. $\left.\{U\}_{k} \eta \in \mathcal{X}_{\partial_{0} \mathscr{C}_{\Omega\left(z_{0}, R\right)}}^{s}\left(\mathscr{C}_{\Omega\left(z_{0}, R\right)}\right)\right)$ for any $U \in \mathcal{X}_{\Sigma_{\mathcal{D}, R}}^{s}\left(\mathscr{C}_{\Omega(z, R)}\right)$ and any function $\eta \in C^{\infty}\left(\mathbb{R}_{+}^{N+1}\right)$ such that $\operatorname{supp}(\eta) \subset B_{\widetilde{\rho}(Z)}(Z)$.

Remark 3.1. It is worth to observe that:

- If $Z \in \Sigma_{\mathcal{D}, R}^{*}$ then $\mathcal{K}^{+}(Z)=[0, \infty), \mathcal{K}^{-}(Z)=(-\infty, 0]$ and $\widetilde{\rho}(Z)=\operatorname{dist}\left(Z, \partial_{B} \mathscr{C}_{\Omega(z, R)}\right)$.

- If $Z \in \mathscr{C}_{\Omega(z, R)}^{\circ} \backslash \Sigma_{\mathcal{D}, R}^{*}$, then $\mathcal{K}^{+}(Z)=\mathcal{K}^{-}(Z)=(-\infty, \infty)$, and in this case $\widetilde{\rho}(Z)=\operatorname{dist}\left(Z, \partial_{0} \mathscr{C}_{\Omega(z, R)}\right)$.

- Thanks to the construction of the cylinder, it is immediate to notice that the number $\widetilde{\rho}(Z)>0$ does not depend on the $y$ variable.

The control of the oscillations of solutions of elliptic problems is usually carried out through integral estimates that mainly rely on a Sobolev-type inequality. Since the extension function solves a degenerate elliptic problem involving a weight (namely, $y^{1-2 s}$ ) that belongs to the Muckenhoupt class $A_{2}$, it is necessary to establish a Sobolev-type inequality dealing with such a type of singular weights. To this aim, we recall the following definition.

Definition 3.2. Given an open subset $D \subset \mathbb{R}^{N}$ and a function $\omega: D \rightarrow \mathbb{R}^{+}$, we say that $\omega$ belongs to the Muckenhoupt class $A_{p}$, with $p>1$ if there exists a constant $C>0$ such that

$$
\sup _{B \subset D}\left(\frac{1}{|B|} \int_{B} \omega^{p}\right)\left(\frac{1}{|B|} \int_{B} \omega^{1-p}\right)^{p-1} \leq C .
$$

Now we can recall the following result.

Theorem 3.1 ([7], Theorem 1.3 and Theorem 1.6). Let $D$ be an open bounded Lipschitz set in $\mathbb{R}^{N}$ and consider $1<p<\infty$ and a weight $\omega \in A_{p}$.

Then, there exist a positive constant $C(D)$ and $\delta>0$ such that for all $u \in H_{0}^{1}(D, \omega)$ and any $1 \leq \sigma \leq \frac{N}{N-1}+\delta$ we have

$$
\|u\|_{L^{\sigma p}(D, \omega d x)} \leq C(D)\|\nabla u\|_{L^{p}(D, \omega d x)},
$$

where $C(D)=c_{\omega} \operatorname{diam}(D)|D|_{\omega}^{\frac{1}{p}\left(\frac{1}{\sigma}-1\right)}$ for a positive constant $c_{\omega}$ depending on $N, p$ and $\omega$.

Moreover for any $x_{0} \in \partial D$ there exist a positive constant $C=C\left(B_{\rho}\left(x_{0}\right)\right)$ and $\delta>0$ such that $1 \leq \sigma \leq \frac{N}{N-1}+\delta$ and any $u \in H^{1}\left(D\left(x_{0}, \rho\right), \omega\right)$ vanishing on $\partial D \cap B_{\rho}\left(x_{0}\right)$ we have

$$
\|u\|_{L^{\sigma p}\left(D\left(x_{0}, \rho\right), \omega d x\right)} \leq C\left(B_{\rho}\right)\|\nabla u\|_{L^{p}\left(\left(D\left(x_{0}, \rho\right), \omega d x\right)\right.},
$$

where $C\left(B_{\rho}\right)=c_{\omega} \rho^{\frac{N}{p}\left(\frac{1}{\sigma}-1\right)+1}$ for a positive constant $c_{\omega}$ depending on $\omega, N, p$ and $\xi$.

We want to apply such a Theorem to domains $D \subsetneq \mathscr{C}_{\Omega} \subset \mathbb{R}_{+}^{N+1}$ so that the correspondent exponent $\sigma$ relies to satisfy $1 \leq \sigma \leq \frac{N+1}{N}$.

As far as the weight is concerned, we set $\omega=y^{1-2 s}$, that, actually, belongs to $A_{2}$. Let us observe that, according to [7, there exists $\varepsilon_{0}>0$ such that (3.9) holds true with $p \geq 2-\varepsilon_{0}$. 
As an immediate consequence of Theorem 3.1 we obtain the following result.

Lemma 3.1. Let $Z \in \Sigma_{\mathcal{D}}^{*}$ and $p \geq 2-\varepsilon_{0}$ for some $\varepsilon_{0}>0$. Then, there exists $\bar{\rho}>0$, such that for any $\rho<\bar{\rho}$ and any $U \in \mathcal{X}_{\Sigma_{\mathcal{D}}}^{s}\left(\mathscr{C}_{\Omega}\right)$ we have

$$
\|U\|_{L^{\sigma p}\left(\mathscr{C}_{\Omega}(Z, \rho), y^{1-2 s} d x d y\right)} \leq c_{s} \rho\left|B_{\rho}\right|_{y^{1-2 s}}^{\frac{1}{p}\left(\frac{1}{\sigma}-1\right)}\|\nabla U\|_{L^{p}\left(\mathscr{C}_{\Omega}(Z, \rho), y^{1-2 s} d x d y\right)},
$$

with $1 \leq \sigma \leq \frac{N+1}{N}+\delta$ for some $\delta>0$ and $c_{s}$ depending on $N, p$ and the weight $y^{1-2 s}$.

Although Theorem 1.1 has been stated for Lipschitz domains, following [10, we might prove most of the results in this section under more general hypotheses on $\partial \Omega$. Then, we relax the smoothness hypotheses on $\partial \Omega$ and establish inequality (3.10) for functions in $\mathcal{X}_{\Sigma_{\mathcal{D}, R}}^{s}\left(\mathscr{C}_{\Omega(z, R)}\right)$ and, given some point $Z \in \mathscr{C}_{\Omega(z, R)}^{\circ} \backslash \Sigma_{\mathcal{D}, R}^{*}$, also for functions in $H^{1}\left(\mathscr{C}_{\Omega}(Z, \rho), y^{1-2 s} d x d y\right)$ vanishing on suitable sets.

Definition 3.3. Given $p \geq 2-\varepsilon_{0}$ for some $\varepsilon_{0} \in(0,1)$ and an open bounded set $A$, we define $\mathcal{F}\left(\beta_{s}, A\right)$ as the family of sets $B \subset \bar{A}$ such that, for any $U \in H^{1}\left(A, y^{1-2 s} d x d y\right)$ vanishing on $B$,

$$
\|U\|_{L^{\sigma p}\left(A, y^{1-2 s} d x d y\right)} \leq \beta_{s} \operatorname{diam}(A)|A|_{y^{1-2 s}}^{\frac{1}{p}\left(\frac{1}{\sigma}-1\right)}\|\nabla U\|_{L^{p}\left(A, y^{1-2 s} d x d y\right)},
$$

for some $\beta_{s}>0$ depending on $N, p$ and the weight $y^{1-2 s}$, and $1 \leq \sigma \leq \frac{N+1}{N}+\delta$ for some $\delta>0$.

With this scheme in mind, we focus first on finding bounds for solutions to (3.4) in terms of the data of the problem.

Theorem 3.2. Let $u$ be a solution to $\overline{P^{s}}$ with $f \in L^{p}(\Omega), p>\frac{N}{2 s}$. Then, there exists a positive constant $C=C\left(N, s,\left|\Sigma_{\mathcal{D}}\right|\right)$ such that

$$
\|u\|_{L^{\infty}(\Omega)} \leq C\|f\|_{L^{p}(\Omega)}|\Omega|^{\frac{2 s}{N}-\frac{1}{p}} .
$$

In the proof of Theorem 3.2 we make use of the following technical result.

Lemma 3.2 ([8], Lemma B.1). Let $\varphi(k)$ be a nonnegative and nonincreasing function defined for $k \geq k_{0}$ such that

$$
\varphi(h) \leq \frac{C_{0}}{(h-k)^{a}} \varphi^{b}(k), \quad k<h,
$$

where $C_{0}, a, b$ are positive constants with $b>1$. Then, $\varphi\left(k_{0}+d\right)=0$, with $d^{a}=2^{\frac{a b}{b-1}} C_{0}\left|\varphi\left(k_{0}\right)\right|^{b-1}$.

Proof of Theorem 3.2. Here we just prove the upper bound, being the lower one completely analogous. Let us take $k \geq 0, U(x, y)=E_{s}[u(x)]$ and $\psi=(U-k)_{+} \in \mathcal{X}_{\Sigma_{\mathcal{D}}}^{s}\left(\mathscr{C}_{\Omega}\right)$ as a test function in (2.2). Using the trace inequality (2.6) together with the Hölder inequality, we get

$$
\begin{aligned}
\kappa_{s} \int_{\mathscr{C}_{\Omega}} y^{1-2 s} \nabla U \nabla \psi d x d y & =\kappa_{s} \int_{A_{+}^{*}(k)} y^{1-2 s}|\nabla U|^{2} d x d y=\int_{A_{+}(k)}(U(x, 0)-k) f(x) d x \\
& \leq\left(\int_{A_{+}(k)}|f|^{2} d x\right)^{\frac{1}{2}}\left(C_{\mathcal{D}}^{-1}\left|A_{+}(k)\right|^{\frac{2 s}{N}} \int_{A_{+}^{*}(k)} y^{1-2 s}|\nabla U|^{2} d x d y\right)^{\frac{1}{2}} .
\end{aligned}
$$

Thus,

$$
\int_{A_{+}^{*}(k)} y^{1-2 s}|\nabla U|^{2} d x d y \leq C_{\mathcal{D}}^{-1} \kappa_{s}^{-2}\left|A_{+}(k)\right|^{\frac{2 s}{N}} \int_{A_{+}(k)}|f|^{2} d x \leq \frac{\|f\|_{L^{p}(\Omega)}^{2}\left|A_{+}(k)\right|^{1-\frac{2}{p}+\frac{2 s}{N}}}{C_{\mathcal{D}} \kappa_{s}^{2}},
$$

and applying the trace inequality (2.6) to the left-hand side of (3.12) we get for any $h>k$,

$$
(h-k)^{2}\left|A_{+}(h)\right|^{\frac{2}{2_{s}^{*}}} \leq\left(\int_{A_{+}(k)}|U(x, 0)-k|^{2_{s}^{*}} d x\right)^{\frac{2}{2_{s}^{*}}} .
$$

Thus we deduce

$$
(h-k)^{2}\left|A_{+}(h)\right|^{\frac{2}{2_{s}^{*}}} \leq \frac{\|f\|_{L^{p}(\Omega)}^{2}}{\left(C_{\mathcal{D}} \kappa_{s}\right)^{2}}\left|A_{+}(k)\right|^{1-\frac{2}{p}+\frac{2 s}{N}},
$$


and setting $\varphi(h)=\left|A_{+}(h)\right|$, it follows that

$$
\varphi(h) \leq \frac{\|f\|_{L^{p}(\Omega)}^{2^{*}}}{\left(C_{\mathcal{D}} \kappa_{s}\right)^{2}} \frac{\varphi^{\left(1-\frac{2}{p}+\frac{2 s}{N}\right) \frac{2_{s}^{*}}{2}}(k)}{(h-k)^{2_{s}^{*}}} .
$$

Applying now Lemma 3.2 with $a=2_{s}^{*}$ and $b=\left(1-\frac{2}{p}+\frac{2 s}{N}\right) \frac{2_{s}^{*}}{2}>1$, we find $\left|\varphi\left(k_{0}+d\right)\right|=0$ with $d=C\left(N, s,\left|\Sigma_{\mathcal{D}}\right|\right)\|f\|_{L^{p}(\Omega)}\left|\varphi\left(k_{0}\right)\right|^{\frac{b-1}{a}}$, and $\frac{b-1}{a}=\frac{2 s}{N}-\frac{1}{p}$, i.e.

$$
U(x, 0) \leq k_{0}+C\left(N, s,\left|\Sigma_{\mathcal{D}}\right|\right)\|f\|_{L^{p}(\Omega)}\left|A_{+}\left(k_{0}\right)\right|^{\frac{2 s}{N}-\frac{1}{p}} \quad \text { a.e. in } \Omega,
$$

for any $k_{0} \geq 0$, and we conclude $u(x) \leq C\left(N, s,\left|\Sigma_{\mathcal{D}}\right|\right)\|f\|_{L^{p}(\Omega)}|\Omega|^{\frac{2 s}{N}-\frac{1}{p}} \quad$ a.e. in $\Omega$.

Let $v(x)$ be the solution to (3.4) and $V(x, y)=E_{s}[v(x)]$ the solution to (3.6). Since the function $(V-k)_{+} \in \mathcal{X}_{\Sigma_{\mathcal{D}}}^{s}\left(\mathscr{C}_{\Omega}\right)$ for any $k \geq 0$, repeating the proof above we deduce that $\forall z \in \Omega$

$$
\|v(x)\|_{L^{\infty}(\Omega(z, R))} \leq C\left(N, s,\left|\Sigma_{\mathcal{D}}\right|\right)\|f\|_{L^{p}(\Omega)}|\Omega(z, R)|^{\frac{2 s}{N}-\frac{1}{p}} .
$$

Now we turn our attention to the study of the behavior of solutions to the homogeneous problem (3.7).

Lemma 3.3 (Caccioppoli inequality). Assume that $z_{0} \in \bar{\Omega}$ and $R>0$ and suppose that the function $W \in \mathcal{X}_{\Sigma_{\mathcal{D}, R}}^{s}\left(\mathscr{C}_{\Omega\left(z_{0}, R\right)}\right)$ is a solution to problem (3.7). Then, for any $Z \in \mathscr{C}_{\Omega\left(z_{0}, R\right)}^{\circ}$ and $0<\rho<r<$ $\widetilde{\rho}(Z)$, we have that there exists $C>0$ such that

$$
\int_{\mathscr{C}_{\Omega\left(z_{0}, R\right)}(Z, \rho)} y^{1-2 s}|\nabla W|^{2} d x d y \leq \frac{C}{(r-\rho)^{2}} \int_{\mathscr{C}_{\Omega\left(z_{0}, R\right)}(Z, r)} y^{1-2 s}|W|^{2} d x d y .
$$

Proof. We use $\psi=\eta^{2} W$ as a test function in (3.8), with $\eta \in C^{1}\left(\mathscr{C}_{\Omega\left(z_{0}, R\right)}\right)$ such that it vanishes on $\partial_{L} \mathscr{C}_{\Omega\left(z_{0}, R\right)} \backslash\left(\Sigma_{\mathcal{D}, R}^{*} \cup \Sigma_{\mathcal{N}, R}^{*}\right)$; observe that in particular $\psi \equiv 0$ on $\partial_{L} \mathscr{C}_{\Omega\left(z_{0}, R\right)} \backslash \Sigma_{\mathcal{N}, R}^{*}$, so that we have that

$$
\begin{gathered}
\int_{\mathscr{C}_{\Omega\left(z_{0}, R\right)}} y^{1-2 s} \eta^{2}|\nabla W|^{2} d x d y=-2 \int_{\mathscr{C}_{\Omega\left(z_{0}, R\right)}} y^{1-2 s}\langle\eta \nabla W, W \nabla \eta\rangle d x d y \\
\leq 2\left(\frac{1}{2 \varepsilon} \int_{\mathscr{C}_{\Omega\left(z_{0}, R\right)}} y^{1-2 s}|\nabla \eta|^{2} W^{2} d x d y+\frac{\varepsilon}{2} \int_{\mathscr{C}_{\Omega\left(z_{0}, R\right)}} y^{1-2 s} \eta^{2}|\nabla W|^{2} d x d y\right),
\end{gathered}
$$

for any $0<\varepsilon<1$. To complete the proof, given $Z \in \mathscr{C}_{\Omega\left(z_{0}, R\right)}^{\circ}$ and $\rho<r<\widetilde{\rho}(Z)$ it is enough to set $\eta$ such that

$$
\eta \equiv 1 \text { in } B_{\rho}(Z), \quad \eta \equiv 0 \text { in } B_{r}^{c}(Z) \quad \text { and } \quad|\nabla \eta| \leq \frac{c}{(r-\rho)} .
$$

and plug into (3.14).

Next we prove the following weighted version of the Poincaré Inequality.

Lemma 3.4. Let $p \geq 2-\varepsilon_{0}$ for some $0<\varepsilon_{0}<1$ and $U \in \mathcal{X}^{s}\left(\mathscr{C}_{\Omega}\right)$ such that $\{U=0\} \in \mathcal{F}(\beta, A)$ for $A \subset \overline{\mathscr{C}}_{\Omega}$. Then $\exists \beta_{s}=\beta_{s}\left(N, p, y^{1-2 s}\right)>0$ such that

$$
\int_{A} y^{1-2 s}|U|^{p} d x d y \leq \beta_{s}^{p}[\operatorname{diam}(A)]^{p}|A|_{y^{1-2 s}}^{\left(\frac{1}{\sigma}-1\right)}|\{(x, y) \in A: U \neq 0\}|_{y^{1-2 s}}^{\frac{1}{\sigma^{\prime}}} \int_{A} y^{1-2 s}|\nabla U|^{p} d x d y
$$

and

$$
\int_{A_{+}^{*}(k, r)} y^{1-2 s}|U-k|^{2} d x d y \leq \beta_{s}^{2} r^{2}\left|B_{r}\right|_{y^{1-2 s}}^{\frac{1}{\sigma}-1}\left|A_{+}^{*}(k, r)\right|_{y^{1-2 s}}^{\frac{1}{\sigma^{\prime}}} \int_{A_{+}^{*}(k, r)} y^{1-2 s}|\nabla U|^{2} d x d y,
$$

with $1 \leq \sigma \leq \frac{N+1}{N}+\delta$ for some $\delta>0$. 
Proof. In fact, (3.15) is consequence of (3.9) and the Hölder inequality.

As far as (3.16) is concerned, we follows [10, Theorem 6.1]: given $U \in \mathcal{X}^{s}\left(\mathscr{C}_{\Omega\left(z_{0}, R\right)}\right)$, let us consider the function $t_{k}^{+}(U)=(U-k)_{+}$that belongs to $\mathcal{X}^{s}\left(\mathscr{C}_{\Omega\left(z_{0}, R\right)}\right)$ for any $k \in \mathbb{R}$. Moreover, if $U \in \mathcal{X}_{\Sigma_{\mathcal{D}, R}}^{s}\left(\mathscr{C}_{\Omega\left(z_{0}, R\right)}\right)$ then $t_{k}^{+}(U) \in \mathcal{X}_{\Sigma_{\mathcal{D}, R}}^{s}\left(\mathscr{C}_{\Omega\left(z_{0}, R\right)}\right)$ for any $k \geq 0$. Then, applying (3.11) to $(U-k)_{+}$with $p=2$, (3.16) follows.

A direct consequence of Lemma 3.4 is the following result.

Lemma 3.5. Given $z_{0} \in \bar{\Omega}$ and $R>0$, let $U \in \mathcal{X}^{s}\left(\mathscr{C}_{\Omega\left(z_{0}, R\right)}\right)$. Then, for any $Z \in \mathscr{C}_{\Omega\left(z_{0}, R\right)}^{\circ}$ and $0<r<\bar{\rho}(Z)$, there exist $\varepsilon_{0} \in(0,1)$ and $\beta_{s}=\beta_{s}\left(N, p, y^{1-2 s}\right)>0$ such that

$$
(h-k)^{2}\left|A_{+}^{*}(h, r)\right|_{y^{1-2 s}}^{\frac{2}{q}} \leq \beta_{s}^{2} r^{2}\left|B_{r}\right|_{y^{1-2 s}}^{2\left(\frac{1}{q}-\frac{1}{p}\right)}\left|A_{+}^{*}(k, r)-A_{+}^{*}(h, r)\right|_{y^{1-2 s}}^{\frac{2}{p}-1} \int_{A_{+}^{*}(k, r)} y^{1-2 s}|\nabla U|^{2} d x d y,
$$

with $h>k, q=\frac{N+1}{N}\left(2-\varepsilon_{0}\right)$ and $p=2-\varepsilon_{0}$.

Proof. Given $U \in \mathcal{X}^{s}\left(\mathscr{C}_{\Omega\left(z_{0}, R\right)}\right)$ and $h>k$, let $t_{h, k}^{+}(U)=\{U\}^{h}-\{U\}^{k}$. Note that $t_{h, k}^{+}(U) \in$ $\mathcal{X}^{s}\left(\mathscr{C}_{\Omega\left(z_{0}, R\right)}\right)$ for any $k \in \mathbb{R}$. Moreover, if $U \in \mathcal{X}_{\Sigma_{\mathcal{D}, R}^{s}}^{s}\left(\mathscr{C}_{\Omega\left(z_{0}, R\right)}\right)$ then $t_{h, k}^{+}(U) \in \mathcal{X}_{\Sigma_{\mathcal{D}, R}}^{s}\left(\mathscr{C}_{\Omega\left(z_{0}, R\right)}\right)$ for any $h>k \geq 0$. Thus, we use Lemma 3.4 with $\sigma=\frac{N+1}{N}$ and $p=2-\varepsilon_{0}$ so that taking $q=\sigma p=\frac{N+1}{N}\left(2-\varepsilon_{0}\right)$ we obtain,

$$
\left(\int_{\mathscr{C}_{\Omega\left(z_{0}, R\right)}(Z, r)} \underset{y^{1-2 s}}{ }\left|t_{h, k}^{+}(U)\right|^{q} d x d y\right)^{\frac{1}{q}} \leq \beta_{s} r\left|B_{r}\right|_{y^{1-2 s}}^{\frac{1}{q}-\frac{1}{p}}\left(\int_{A_{+}^{*}(k, r)-A_{+}^{*}(h, r)} y^{1-2 s}|\nabla U|^{p} d x d y .\right)^{\frac{1}{p}} .
$$

At one hand, it is immediate that

$$
(h-k)^{2}\left|A_{+}^{*}(h, r)\right|_{y^{1-2 s}}^{\frac{2}{q}} \leq\left(\int_{\mathscr{C}_{\Omega\left(z_{0}, R\right)}(Z, r)} y^{1-2 s}\left|t_{h, k}^{+}(U)\right|^{q} d x d y\right)^{\frac{2}{q}} .
$$

On the other hand, thanks to Hölder inequality

$$
\left(\int_{A_{+}^{*}(k, r)-A_{+}^{*}(h, r)} y^{1-2 s}|\nabla U|^{p} d x d y\right)^{\frac{2}{p}} \leq\left|A_{+}^{*}(k, r)-A_{+}^{*}(h, r)\right|_{y^{1-2 s}}^{\frac{2}{p}-1} \int_{A_{+}^{*}(k, r)} y^{1-2 s}|\nabla U|^{2} d x d y .
$$

Thus (3.17) follows by gathering together (3.18), (3.19) and (3.20).

Following [10, Theorem 8.1], we show the next result.

Theorem 3.3. Let $z_{0} \in \bar{\Omega}, R>0$, and let $W \in \mathcal{X}_{\Sigma_{\mathcal{D}, R}}^{s}\left(\mathscr{C}_{\Omega\left(z_{0}, R\right)}\right)$ be a solution to the homogeneous problem (3.7). Then, for any $Z \in \mathscr{C}_{\Omega\left(z_{0}, R\right)}^{\circ}, 0<\ell<1$ and $0<r<\min \{\widetilde{\rho}(Z), \bar{\rho}(Z)\}$, there exists a positive constant $\Lambda=\Lambda(\ell)$ such that

$\left|A_{+}^{*}(k+\ell d, r-\ell r)\right|=0, \quad$ with $k \in \mathcal{K}^{+}(Z) \quad$ and $\quad\left|A_{-}^{*}(k-\ell d, r-\ell r)\right|=0, \quad$ with $k \in \mathcal{K}^{-}(Z)$, where

$$
d^{2} \geq \frac{1}{\Lambda(\ell)\left|B_{r}\right|_{y^{1-2 s}}} \int_{A_{+}^{*}(k, r)} y^{1-2 s}|W-k|^{2} d x d y .
$$

In the proof of Theorem 3.3 we make use of the following technical result.

Lemma 3.6 (8], Lemma C.7). Assume that $\varphi(k, \rho)$ is a nonnegative function defined for $k \geq k_{0}$ and $0<\rho \leq r_{0}$ which is nonincreasing with respect to $k$, nondecreasing with respect to $\rho$ and such that

$$
\varphi(h, \rho) \leq \frac{C_{0}}{(h-k)^{\alpha}(r-\rho)^{\gamma}} \varphi^{\mu}(k, r), \quad k<h, \rho<r \leq r_{0},
$$

where $C, \alpha, \beta, \gamma$ are positive constants with $\mu>1$. Then there exist $\ell \in(0,1)$ and $d>0$ such that $\varphi\left(k_{0}+\ell d, r_{0}(1-\ell)\right)=0$, with

$$
d^{\alpha}=C_{0} \frac{2^{(\alpha+\gamma) \frac{\mu}{\mu-1}}\left[\varphi\left(k_{0}, r_{0}\right)\right]^{\mu-1}}{\ell^{\alpha+\gamma} r_{0}^{\gamma}}
$$


Proof of Theorem 3.3. Given $z_{0} \in \bar{\Omega}, k_{0} \in \mathcal{K}^{+}\left(z_{0}\right)$ and $k \geq k_{0}$, let us define

$$
i(k, \rho)=\int_{A^{*}(k, \rho)} y^{1-2 s}|W-k|^{2} d x d y \quad \text { and } \quad a(k, \rho)=\left|A_{+}^{*}(k, \rho)\right|_{y^{1-2 s}} .
$$

Observe that for $h>k$ we have

$$
(h-k)^{2}\left|A_{+}^{*}(h, \rho)\right|_{y^{1-2 s}} \leq \int_{A_{+}^{*}(k, r)} y^{1-2 s}|W-k|^{2} d x d y .
$$

Assume that $Z \in \Sigma_{\mathcal{D}, R}^{*} \cap \mathscr{C}_{\Omega\left(z_{0}, R\right)}$ and let $0<r_{0}<\min \{\widetilde{\rho}(Z), \bar{\rho}(Z)\}$. Then, due to Lemma 3.3 and Lemma 3.4 for any $r_{0}(1-\ell) \leq \rho<r \leq r_{0}$ and $h>k$, we have

$$
\begin{aligned}
& \int_{A_{+}^{*}(h, \rho)}^{y^{1-2 s}}|W-h|^{2} d x d y \leq K_{\mathscr{C}_{\Omega}(\rho)}\left(\int_{A_{+}^{*}(h, \rho)}^{y^{1-2 s}}|\nabla W|^{2} d x d y\right)\left|A_{+}^{*}(h, \rho)\right|_{y^{1-2 s}}^{\frac{1}{\sigma^{\prime}}} \\
& \leq K_{\mathscr{C}_{\Omega}(\rho)}\left(\int_{A_{+}^{*}(k, \rho)} y^{1-2 s}|\nabla W|^{2} d x d y\right)\left|A_{+}^{*}(k, \rho)\right|_{y^{1-2 s}}^{\frac{1}{\sigma^{\prime}}}
\end{aligned}
$$

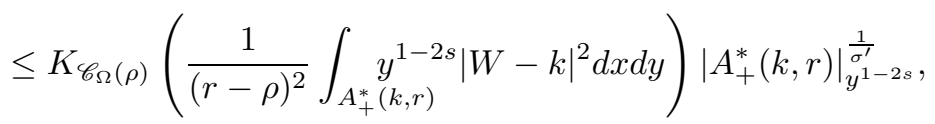

where $K_{\mathscr{C}_{\Omega}(r)}=\beta_{s}^{2} r^{2}\left|B_{r}\right|_{y^{1-2 s}}^{\frac{1}{\sigma}-1}$, with $\beta_{s}=\beta_{s}\left(N, y^{1-2 s}, \partial \Omega\right)>0$ and $1 \leq \sigma \leq \frac{N+1}{N}+\delta$ for some $\delta>0$.

Assume, on the contrary, that $Z_{0} \in \mathscr{C}_{\Omega\left(z_{0}, R\right)}^{\circ} \backslash \Sigma_{\mathcal{D}, R}^{*}$. Recalling (3.2), let $\Lambda=\Lambda(\ell)>0$ satisfying

$$
\frac{\Lambda}{\zeta_{s}(1-\ell)^{N+2(1-s)}} \leq(1-\lambda) \quad \text { for some } \lambda \in(0,1) \text {. }
$$

Therefore, given $h \geq k_{0}$ and $(1-\ell) r_{0} \leq \rho \leq r_{0}$, we find

$$
\begin{aligned}
& \left|A_{+}^{*}(h, \rho)\right|_{y^{1-2 s}} \leq\left|A_{+}^{*}\left(k_{0}, r_{0}\right)\right|_{y^{1-2 s}} \leq\left|\mathscr{C}_{\Omega\left(z_{0}, R\right)}\left(Z, r_{0}\right)\right|_{y^{1-2 s}} \leq\left|B_{r_{0}}(Z)\right|_{y^{1-2 s}} \\
& \leq \frac{\left|B_{\rho}(Z)\right|_{y^{1-2 s}}}{(1-\ell)^{N+2(1-s)}} \leq \frac{\Lambda\left|\mathscr{C}_{\Omega\left(z_{0}, R\right)}(Z, \rho)\right|_{y^{1-2 s}}}{\zeta_{s}(1-\ell)^{N+2(1-s)}} \leq(1-\lambda)\left|\mathscr{C}_{\Omega\left(z_{0}, R\right)}(Z, \rho)\right|_{y^{1-2 s}} .
\end{aligned}
$$

Using Lemma 3.3 and Lemma 3.4 we deduce that (3.23) holds true.

As a consequence, for any $Z \in \mathscr{C}_{\Omega\left(z_{0}, R\right)}^{\circ}$,

$$
i(h, \rho) \leq \frac{K_{\mathscr{C}_{\Omega}}(\rho)}{(r-\rho)^{2}} i(k, r)[a(k, r)]^{\frac{1}{\sigma^{\prime}}}, \quad r_{0}(1-\ell) \leq \rho<r \leq r_{0}, \text { and } h>k \geq k_{0}
$$

with $k_{0} \in \mathcal{K}^{+}(Z)$ satisfying (3.3). Moreover, since $\left|B_{\mu r}\right|_{y^{1-2 s}}=\mu^{N+2(1-s)}\left|B_{r}\right|_{y^{1-2 s}}$, we have that $K_{\mathscr{C}_{\Omega}(\mu r)}=\mu^{\varsigma} K_{\mathscr{C}_{\Omega}\left(r_{0}\right)}$, where $\varsigma=2+\left(\frac{1}{\sigma}-1\right)(N+2(1-s))$.

If we let $1<\sigma \leq 1+\frac{2}{N-2 s}$ (so that $\varsigma>0$ ) then $K_{\mathscr{C}_{\Omega}(r)} \leq K_{\mathscr{C}_{\Omega}\left(r_{0}\right)}$ for any $0<r<r_{0}$. Hence, from (3.24), we obtain

$$
i(h, \rho) \leq \frac{K_{\mathscr{C}_{\Omega}\left(r_{0}\right)}}{(r-\rho)^{2}} i(k, r)[a(k, r)]^{\frac{1}{\sigma^{\top}}}, \quad \rho<r \leq r_{0}, h>k \geq k_{0},
$$

with $K_{\mathscr{C}_{\Omega}\left(r_{0}\right)}=\beta_{s}^{2} r_{0}^{2}\left|B_{r_{0}}\right|_{y^{1-2 s}}^{\frac{1}{\sigma}-1}$. We set now $\xi+1=\theta \xi$ and $\frac{\xi}{\sigma^{\prime}}=\theta$, so that $\theta=\frac{1}{2}+\sqrt{\frac{1}{4}+\frac{1}{\sigma^{\prime}}}>1$ turns out to be the unique positive solution to the equation $\theta^{2}-\theta-\frac{1}{\sigma^{\prime}}=0$. Assume in addition that the constant $\Lambda$ satisfies

$$
\Lambda^{\frac{\theta}{2}} \leq \frac{\ell^{\xi+1}}{\beta_{s}^{\xi} 2^{(\xi+1) \frac{\theta}{\theta-1}}}
$$

From (3.22) and (3.25), we obtain

$$
|i(h, \rho)|^{\xi}|a(h, \rho)| \leq \frac{K_{\mathscr{C}_{\Omega}\left(r_{0}\right)}^{\xi}}{(r-\rho)^{2 \xi}(h-k)^{2}}|i(k, r)|^{\xi+1}|a(k, r)|^{\frac{\xi}{\sigma^{\prime}}} .
$$


Then, taking $\varphi(k, \rho)=|i(k, \rho)|^{\xi}|a(k, \rho)|$, it follows that $\varphi$ satisfies

$$
\varphi(h, \rho) \leq \frac{K_{\mathscr{C}_{\Omega}\left(r_{0}\right)}^{\xi}}{(r-\rho)^{\xi \xi}(h-k)^{2}} \varphi^{\theta}(k, r), \quad h>k \geq k_{0}, \rho<r \leq r_{0} .
$$

Using Lemma 3.6 with $\alpha=2, \mu=\theta, \gamma=2 \xi$, we deduce that exist $d_{0}>0$ and $\ell \in(0,1)$ such that

$$
\varphi\left(k_{0}+\ell d_{0}, r_{0}(1-\ell)\right)=0,
$$

for any $k_{0} \in \mathcal{K}^{+}(Z)$ satisfying (3.3), $0<r_{0}<\min \{\widetilde{\rho}(Z), \bar{\rho}(Z)\}$ and $d_{0}$ such that

$$
d_{0}=\frac{2^{\frac{(\xi+1) \theta}{\theta-1}}}{\ell^{\xi+1}} \frac{K_{\mathscr{C}_{\Omega}\left(r_{0}\right)}^{\xi / 2}\left[\varphi\left(k_{0}, r_{0}\right)\right]^{\frac{\theta-1}{2}}}{r_{0}^{\xi}} \geq\left(\frac{1}{\Lambda\left|B_{r_{0}}\right| y^{1-2 s}} \int_{A_{+}^{*}\left(k, r_{0}\right)} y^{1-2 s}\left|W-k_{0}\right|^{2} d x d y\right)^{\frac{1}{2}} .
$$

Since $\left|A_{+}^{*}\left(k_{0}+\ell d_{0}, r_{0}(1-\ell)\right)\right|_{y^{1-2 s}}=0$ implies $\left|A_{+}^{*}\left(k_{0}+\ell d_{0}, r_{0}(1-\ell)\right)\right|=0$ the proof is complete.

The proof on the lower bound follows using the same inequalities on $(W+k)^{-}$and getting the bounds on $\left|A_{-}^{*}\left(k_{0}-\ell d, r_{0}(1-\ell)\right)\right|_{y^{1-2 s}}$.

As a consequence of the above Theorem we get the $L^{\infty}$ bound on $W$.

Corollary 3.1. Let $z_{0} \in \bar{\Omega}, R>0$, and let $W \in \mathcal{X}_{\Sigma_{\mathcal{D}, R}}^{s}\left(\mathscr{C}_{\Omega\left(z_{0}, R\right)}\right)$ be a solution to the homogeneous problem (3.7); consider the set $\mathscr{C}_{\Omega\left(z_{0}, R / 2\right)}^{m}=\mathscr{C}_{\Omega\left(z_{0}, R / 2\right)} \cap\{y<m\}$ with $m>0$. Then, $W \in L^{\infty}\left(\mathscr{C}_{\Omega\left(z_{0}, R / 2\right)}^{m}\right)$ for any $m>0$.

In particular, any solution $w \in H_{\Sigma_{\mathcal{D}, R}}^{s}\left(\Omega\left(z_{0}, R\right)\right)$ of problem (3.5), satisfies $w \in L^{\infty}\left(\Omega\left(z_{0}, R / 2\right)\right)$.

Proof. First, let us prove that $w \in L^{\infty}\left(\Omega\left(z_{0}, R / 2\right)\right)$ with $w$ satisfying problem (3.5). Let $W \in$ $\mathcal{X}_{\Sigma_{\mathcal{D}, R}}^{s}\left(\mathscr{C}_{\Omega\left(z_{0}, R\right)}\right)$ a solution to problem (3.7) and since $\Omega\left(z_{0}, R / 2\right)$ is a bounded set, there exists $Z_{i}=\left(z_{i}, 0\right) \in \mathscr{C}_{\Omega\left(z_{0}, R\right)}^{\circ}, i=1,2, \ldots, M$ such that

$$
\overline{\Omega\left(z_{0}, R / 2\right)}=\left(\bigcup_{i=1}^{M} \mathscr{C}_{\Omega\left(z_{0}, R\right)}^{\circ}\left(Z_{i}, r_{i} / 2\right)\right) \cap\{y=0\},
$$

with $0<r_{i}<\left\{\widetilde{\rho}\left(Z_{i}\right), \bar{\rho}\left(Z_{i}\right)\right\}$. Let $\bar{k}>0$ and $\hat{k}<0$ be such that,

$$
\left|A_{+}^{*}\left(\bar{k}, r_{i}\right)\right| \leq \Lambda\left|\mathscr{C}_{\Omega\left(z_{0}, R\right)}\left(Z_{i}, r_{i}\right)\right|, \quad \text { and } \quad\left|A_{-}^{*}\left(\hat{k}, r_{i}\right)\right| \leq \Lambda\left|\mathscr{C}_{\Omega\left(z_{0}, R\right)}\left(Z_{i}, r_{i}\right)\right|,
$$

for any $i=1,2, \ldots, M$. Then, applying Theorem 3.3 we conclude that, given $X \in \mathscr{C}_{\Omega\left(z_{0}, R\right)}\left(Z_{i}, r_{i}\right)$ for some $i=1,2, \ldots, M$; we have

$$
\kappa_{m}:=\hat{k}-\ell d \leq W(x, y) \leq \kappa_{M}:=\bar{k}+\ell d,
$$

with

$$
d^{2} \geq \frac{1}{\Lambda\left|B_{r}\right|_{y^{1-2 s}}} \int_{\mathscr{C}_{\Omega\left(z_{0}, R\right)}} y^{1-2 s}|W|^{2} d x d y,
$$

for any $0<r<\min _{i=1, \ldots, M} r_{i}$. In particular, by (3.27), the former inequality holds for any point $X=(x, 0)$ with $x \in \overline{\Omega\left(z_{0}, R / 2\right)}$ and we are done.

As $\mathscr{C}_{\Omega\left(z_{0}, R / 2\right)}$ is an unbounded domain, if we repeat the steps above in order to prove that $W \in L^{\infty}\left(\overline{\mathscr{C}}_{\Omega\left(z_{0}, R / 2\right)}\right)$ from (3.28), the numbers $\hat{k}, \bar{k}$ do diverge when considering a covering sequence $\left\{Z_{i}\right\}_{i \in \mathbb{N}}$. Nevertheless, it is clear that given any finite truncation of the extension cylinder, $\mathscr{C}_{\Omega\left(z_{0}, R / 2\right)}^{m}=\mathscr{C}_{\Omega\left(z_{0}, R / 2\right)} \cap\{y<m\}$, there exists a finite covering sequence and hence, we conclude $W \in L^{\infty}\left(\overline{\mathscr{C}_{\Omega\left(z_{0}, R / 2\right)}^{m}}\right)$ for all finite $m>0$.

We focus now on the oscillation of the solutions $W \in \mathcal{X}_{\Sigma_{\mathcal{D}, R}}^{s}\left(\mathscr{C}_{\Omega\left(z_{0}, R\right)}\right)$ to problem (3.7). Let us set

$$
m(\rho)=\inf _{X \in \overline{\mathscr{C}}_{\Omega\left(z_{0}, R\right)}(Z, \rho)} W(X) \quad \text { and } \quad M(\rho)=\sup _{X \in \overline{\mathscr{C}}_{\Omega\left(z_{0}, R\right)}(Z, \rho)} W(X) .
$$


and define the oscillation function as

$$
\omega(\rho):=M(\rho)-m(\rho) .
$$

Our aim is to give some estimates on $\omega(\rho)$ through the following result.

Theorem 3.4. Given $z_{0} \in \bar{\Omega}$ and $R>0$, let $Z \in \mathscr{C}_{\Omega\left(z_{0}, R\right)}^{\circ}$ and let $W \in \mathcal{X}_{\Sigma_{\mathcal{D}, R}}\left(\mathscr{C}_{\Omega\left(z_{0}, R\right)}\right)$ be a solution to the homogeneous problem (3.7). Moreover, given $0<4 \rho<\min \{\widetilde{\rho}(Z), \bar{\rho}(Z)\}$ let $0<\eta<1$ such that,

(i) $(M(4 \rho)-\eta \omega(4 \rho),+\infty) \subset \mathcal{K}^{+}(Z)$

(ii) $\left|A_{+}^{*}(M(4 \rho)-\eta \omega(4 \rho), 2 \rho)\right|_{y^{1-2 s}} \leq \Lambda\left|\mathscr{C}_{\Omega\left(z_{0}, R\right)}(Z, 2 \rho)\right|_{y^{1-2 s}}$,

where $\Lambda$ is determined by (3.21) with $\ell=\frac{1}{2}$. Then, there exists $0<\bar{\eta}<1$ independent from $Z$ and $\rho$ such that,

$$
\omega(\rho) \leq \bar{\eta} \omega(4 \rho)
$$

Proof. Let $Z \in \mathscr{C}_{\Omega\left(z_{0}, R\right)}^{\circ}$ and $0<4 \rho<\min \{\widetilde{\rho}(Z), \bar{\rho}(Z)\}$, let us define the sequence

$$
k_{j}=M(4 \rho)-\eta_{j} \omega(4 \rho), \quad \text { with } \eta_{j}=\frac{1}{2^{j+1}}, j \in \mathbb{N} .
$$

Assume first that $Z \in \mathscr{C}_{\Omega\left(z_{0}, R\right)}^{\circ} \backslash \Sigma_{\mathcal{D}, R}^{*}$ so that $\mathcal{K}^{+}(Z)=(-\infty, \infty)$ and observe that one of the following conditions is satisfied: either

$$
\left|A_{+}^{*}\left(k_{0}, 2 \rho\right)\right|_{y^{1-2 s}} \leq \frac{1}{2}\left|\mathscr{C}_{\Omega\left(z_{0}, R\right)}(Z, 2 \rho)\right|_{y^{1-2 s}} \quad \text { or } \quad\left|A_{-}^{*}\left(k_{0}, 2 \rho\right)\right|_{y^{1-2 s}} \leq \frac{1}{2}\left|\mathscr{C}_{\Omega\left(z_{0}, R\right)}(Z, 2 \rho)\right|_{y^{1-2 s}} .
$$

Assume without loss of generality that $\left|A_{+}^{*}\left(k_{0}, 2 \rho\right)\right| \leq \frac{1}{2}\left|\mathscr{C}_{\Omega\left(z_{0}, R\right)}(Z, 2 \rho)\right|$. As a consequence,

$$
\left|A_{+}^{*}\left(k_{j}, 2 \rho\right)\right| \leq \frac{1}{2}\left|\mathscr{C}_{\Omega\left(z_{0}, R\right)}(Z, 2 \rho)\right| \quad \text { for } \quad j \geq 1 .
$$

On the other hand, if $Z \in \Sigma_{\mathcal{D}, R}^{*}$, we can assume that at least one between $M(4 \rho)$ and $-m(4 \rho)$ is greater than $\frac{1}{2} \omega(4 \rho)$; suppose that $M(4 \rho)>\frac{1}{2} \omega(4 \rho)$. Therefore we have that $k_{j}>0$ for $j \geq 0$.

Then, using Lemma 3.5 with $h=k_{j+1}$ and $k=k_{j}$, we obtain

$$
\left(k_{j+1}-k_{j}\right)^{2}\left|A_{+}^{*}\left(k_{j+1}, 2 \rho\right)\right|_{y^{1-2 s}}^{\frac{2}{q}} \leq \beta_{s}^{2}(2 \rho)^{2}\left|B_{2 \rho}\right|_{y^{1-2 s}}^{2\left(\frac{1}{q}-\frac{1}{p}\right)} \int_{A_{+}^{*}\left(k_{j}, 2 \rho\right)} y^{1-2 s}|\nabla W|^{2} d x d y,
$$

with $p, q$ such that $q=\frac{N+1}{N}\left(2-\varepsilon_{0}\right)$ and $p=2-\varepsilon_{0}$ for a suitable $\varepsilon_{0}>0$.

Moreover, applying Lemma 3.3 to the function $t_{k_{j}}^{+}(W) \in \mathcal{X}_{\Sigma_{\mathcal{D}, R}}^{s}\left(\mathscr{C}_{\Omega(z, R)}\right), j \geq 0$, we find

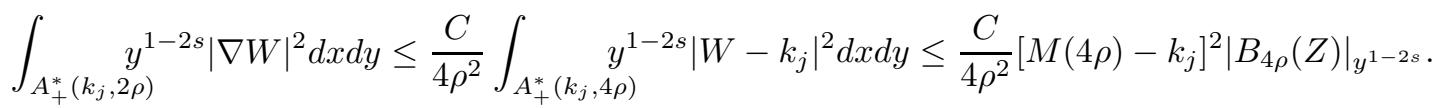

Gathering together the above inequalities we have that

$\left(k_{j+1}-k_{j}\right)^{2}\left|A_{+}^{*}\left(k_{j+1}, 2 \rho\right)\right|_{y^{1-2 s}}^{\frac{2}{q}} \leq C \beta_{s}\left|B_{2 \rho}\right|_{y^{1-2 s}}^{2\left(\frac{1}{q}-\frac{1}{p}\right)+1}\left[M(4 \rho)-k_{j}\right]^{2}\left|A_{+}^{*}\left(k_{j}, 2 \rho\right)-A_{+}^{*}\left(k_{j+1}, 2 \rho\right)\right|_{y^{1-2 s}}^{\frac{2}{p}-1}$, where the constant $C>0$ is the one appearing in the Caccioppoli inequality. Let us define

$$
\varphi(k)=\frac{\left|A_{+}^{*}(k, 2 \rho)\right|_{y^{1-2 s}}}{\left|\mathscr{C}_{\Omega(z, R)}(Z, 2 \rho)\right|_{y^{1-2 s}}},
$$

and note that, by (3.1) and (3.2), we have $\left|B_{2 \rho}\right|_{y^{1-2 s}} \leq \frac{1}{\zeta_{s}}\left|\mathscr{C}_{\Omega(z, R)}(Z, 2 \rho)\right|_{y^{1-2 s}}$. Then, since $2\left(\frac{1}{q}-\frac{1}{p}\right)+1>0$, taking into account that

$$
k_{j+1}-k_{j}=\eta_{j+1} \omega(4 \rho) \quad \text { and } \quad M(4 \rho)-k_{j}=\eta_{j} \omega(4 \rho),
$$

from (3.30) we find

$$
\left|\varphi\left(k_{j+1}\right)\right|^{\frac{2}{q}} \leq \vartheta\left[\varphi\left(k_{j}\right)-\varphi\left(k_{j+1}\right)\right]^{\frac{2}{p}-1} \quad \text { with } \quad \vartheta=\frac{4 C \beta_{s}}{\zeta_{s}^{2\left(\frac{1}{q}-\frac{1}{p}\right)+1}} .
$$


Let us set $\mu=\frac{2}{q} \frac{1}{\frac{2}{p}-1}>0$ and $a=\frac{p}{2-p}$, so that the above inequality turns into

$$
\varphi^{\mu}\left(k_{n}\right) \leq \vartheta^{a}\left[\varphi\left(k_{j}\right)-\varphi\left(k_{j+1}\right)\right], \quad j \geq 0 .
$$

Summing up the above inequality for $j=0,1, \ldots, n$ and noticing that $\varphi\left(k_{j}\right) \geq \varphi\left(k_{n}\right)$ we get

$$
n \varphi^{\mu}\left(k_{n}\right) \leq \vartheta^{a}\left[\varphi\left(k_{0}\right)-\varphi\left(k_{n+1}\right)\right],
$$

and by (3.30), we conclude that

$$
\varphi\left(k_{n}\right) \leq\left(\frac{\vartheta^{a} \varphi\left(k_{0}\right)}{n}\right)^{\frac{1}{\mu}}
$$

Let us set $\bar{n}>0$ such that

$$
\bar{n} \geq\left\lceil\frac{\left(4 C \beta_{s}\right)^{a} \varphi\left(k_{0}\right)}{\zeta_{s}^{\mu-1} \Lambda^{\mu}}\right\rceil,
$$

where $\Lambda$ is determined by (3.3) with $\ell=\frac{1}{2}, \zeta_{s}$ depends on $\zeta$ in (3.1) and the $A_{2}$-constant (see (3.2)), the constant $\beta_{s}$ depends on $N$ and the weight $y^{1-2 s}$ and $C>0$ is an universal constant coming from the Caccioppoli inequality.

Consequently, $\bar{n}$ is independent of $Z$ and $\rho$. Then, by inequality (3.31), we find

$$
\frac{\left|A_{+}^{*}\left(k_{n}, 2 \rho\right)\right|_{y^{1-2 s}}}{\left|\mathscr{C}_{\Omega(z, R)}(Z, 2 \rho)\right|_{y^{1-2 s}}} \leq \Lambda, \quad \forall n \geq \bar{n}
$$

Applying Theorem 3.3 with $k_{\bar{n}}=M(4 \rho)-\eta_{\bar{n}} \omega(4 \rho), r=2 \rho$ and $\ell=\frac{1}{2}$, so that

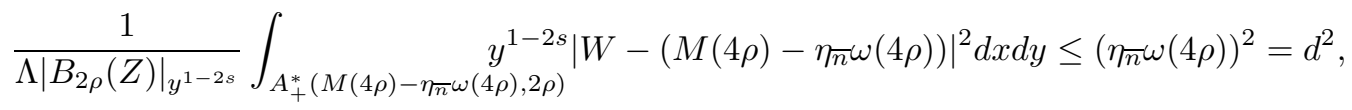

we obtain,

$$
W(X) \leq k+\ell d \leq\left[M(4 \rho)-\eta_{\bar{n}} \omega(4 \rho)\right]+\frac{1}{2} \eta_{\bar{n}} \omega(4 \rho) \leq M(4 \rho)-\frac{1}{2} \eta_{\bar{n}} \omega(4 \rho), \quad \text { a.e. in } \mathscr{C}_{\Omega(z, R)(Z, \rho)} .
$$

As a consequence,

$$
\omega(\rho)=M(\rho)-m(\rho) \leq M(\rho)-m(4 \rho) \leq\left[M(4 \rho)-\frac{1}{2} \eta_{\bar{n}} \omega(4 \rho)\right]-m(4 \rho) \leq\left(1-\frac{1}{2} \eta_{\bar{n}}\right) \omega(4 \rho),
$$

and we deduce (3.29) by choosing $\bar{\eta}=\left(1-\eta_{\bar{n}+1}\right)$.

The next result gives an estimate on the growth of the oscillation.

Theorem 3.5. Given $z_{0} \in \bar{\Omega}$ and $R>0$, let $W \in \mathcal{X}_{\Sigma_{\mathcal{D}, R}}^{s}\left(\mathscr{C}_{\Omega\left(z_{0}, R\right)}\right)$ be a solution to the homogeneous problem (3.7). Then, there exist $0<\mathcal{H}<1$ and $0<\tau<\frac{1}{2}$ such that for any $Z \in \mathscr{C}_{\Omega\left(z_{0}, R\right)}^{\circ}$ there exists $\delta(Z)>0$ such that

$$
\omega(\rho)=\sup _{X \in \overline{\mathscr{C}}_{\Omega\left(z_{0}, R\right)}(Z, \rho)} W(X)-\inf _{X \in \overline{\mathscr{C}}_{\Omega\left(z_{0}, R\right)}(Z, \rho)} W(X) \leq \mathcal{H} \rho^{\tau},
$$

for any $0<\rho<\delta(Z)$.

Proof. Let $r(Z)=\min \{\widetilde{\rho}(Z), \bar{\rho}(Z)\}$, by Theorem 3.4, inequality (3.29) holds true for any $\rho<r(Z) / 4$. Take $\tau, M$ positive such that $4^{\tau} \bar{\eta}=a<1$ and $\omega(\rho) \leq M \rho^{\tau}$ for $\frac{r(Z)}{4} \leq \rho<r(Z)$. Then, again by (3.29), we have that

$$
\omega(\rho) \leq \bar{\eta} 4^{\tau} M \rho^{\tau},
$$

for $\frac{r(Z)}{4^{2}} \leq \rho<\frac{r(Z)}{4}$. In general, if $\frac{r(Z)}{4^{i+1}} \leq \rho<\frac{r(Z)}{4^{i}}$ for some $i \in \mathbb{N}$, we deduce that $\omega(\rho) \leq$ $\left(\bar{\eta} 4^{\tau}\right)^{i} M \rho^{\tau}$. Letting $\bar{i}$ large enough such that $\mathcal{H}=M a^{\bar{i}}<1$, we obtain $\omega(\rho) \leq \mathcal{H} \rho^{\tau}$ for any $\rho<\delta(Z)=\frac{r(Z)}{4^{\bar{\imath}}}$. On the other hand, since we have chosen $\tau>0$ such that $4^{\tau} \bar{\eta}<1$ and, by Theorem 3.4, $\bar{\eta}=1-\eta_{\bar{n}+1}$ for some $\bar{n} \geq 0$ independent from $Z$ and $\rho$, it follows that

$$
\tau<\frac{1}{2} \log _{2}\left(\frac{2^{\bar{n}+2}}{2^{\bar{n}+2}-1}\right)<\frac{1}{2} .
$$


Before proving Theorem 1.1 let us observe the following:

(i) if $z_{0} \in \Omega$, then there exist $R>0$ sufficiently small such that $\Sigma_{\mathcal{D}, R}=\Sigma_{\mathcal{N}, R}=\emptyset$ and $\widetilde{\rho}(Z)=\operatorname{dist}\left(Z, \partial_{L} \mathscr{C}_{\Omega\left(z_{0}, R\right)}\right)$ for any $z \in \mathscr{C}_{\Omega\left(z_{0}, R\right)}$.

(ii) if $z_{0} \in \Sigma_{\mathcal{D}} \backslash \Gamma$, then there exist $R>0$ such that $\Sigma_{\mathcal{N}, R}=\emptyset$. Hence $\widetilde{\rho}(Z)=\operatorname{dist}\left(Z, \partial_{B} \mathscr{C}_{\Omega\left(z_{0}, R\right)}\right)$ for any $Z \in \Sigma_{\mathcal{D}, R}^{*}$ and $\widetilde{\rho}(Z)=\operatorname{dist}\left(Z, \partial_{0} \mathscr{C}_{\Omega\left(z_{0}, R\right)}\right)$ for any $Z \in \mathscr{C}_{\Omega\left(z_{0}, R\right)}^{\circ} \backslash \Sigma_{\mathcal{D}, R}^{*}$.

(iii) if $z_{0} \in \Sigma_{\mathcal{N}}$, then there exist $R>0$ such that $\Sigma_{\mathcal{D}, R}=\emptyset$. Hence we have $\widetilde{\rho}(Z)=\operatorname{dist}\left(Z, \partial_{B} \mathscr{C}_{\Omega\left(z_{0}, R\right)}\right)$ for any $Z \in \mathscr{C}_{\Omega\left(z_{0}, R\right)}^{\circ}$.

(iv) if $z_{0} \in \Gamma$ then $\forall R>0$ both $\Sigma_{\mathcal{D}, R} \neq \emptyset$ and $\Sigma_{\mathcal{N}, R} \neq \emptyset$ and hence $\widetilde{\rho}(Z)=\operatorname{dist}\left(Z, \partial_{B} \mathscr{C}_{\Omega\left(z_{0}, R\right)}\right)$ for any $Z \in \Sigma_{\mathcal{D}, R}^{*}$ and $\widetilde{\rho}(Z)=\operatorname{dist}\left(Z, \partial_{0} \mathscr{C}_{\Omega\left(z_{0}, R\right)}\right)$ for any $Z \in \mathscr{C}_{\Omega\left(z_{0}, R\right)}^{\circ} \backslash \Sigma_{\mathcal{D}, R}^{*}$.

Now, consider $\overline{\mathscr{C}}_{\Omega\left(z_{0}, R / 2\right)} \subset \mathscr{C}_{\Omega(z, R)}$ if $z \in \Omega$ and $\overline{\mathscr{C}}_{\Omega\left(z_{0}, R / 2\right)} \subset \mathscr{C}_{\Omega(z, R)}^{\circ}$ if $z \in \partial \Omega$.

Thus we deduce that:

(i) if $z \in \Omega$, then $\widetilde{\rho}(Z)=\operatorname{dist}\left(Z, \partial_{L} \mathscr{C}_{\Omega(z, R)}\right) \geq \widetilde{\rho}>0$ for any $Z \in \overline{\mathscr{C}}_{\Omega(z, R / 2)}$ and some positive $\tilde{\rho}$.

(ii) if $z \in \Sigma_{\mathcal{D}} \backslash \Gamma$, then $\widetilde{\rho}(Z)=\widetilde{\rho}>0$ for some positive $\widetilde{\rho}$ for any $Z \in \Sigma_{\mathcal{D}, R / 2}^{*}$ and $\widetilde{\rho}(Z)=$ $\operatorname{dist}\left(Z, \Sigma_{\mathcal{D}, R / 2}^{*}\right)$ for any $Z \in \overline{\mathscr{C}}_{\Omega(z, R / 2)} \backslash \Sigma_{\mathcal{D}, R / 2}^{*}$.

(iii) if $z \in \Sigma_{\mathcal{N}}$, then $\widetilde{\rho}(Z)=\operatorname{dist}\left(Z, \partial_{B} \mathscr{C}_{\Omega(z, R)}\right) \geq \widetilde{\rho}>0$ for any $Z \in \overline{\mathscr{C}}_{\Omega(z, R / 2)}$ and some positive $\widetilde{\rho}$.

(iv) if $z \in \Gamma$ then $\widetilde{\rho}(Z)=\widetilde{\rho}>0$ for some positive $\widetilde{\rho}$ for any $Z \in \Sigma_{\mathcal{D}, R / 2}^{*}$ and $\widetilde{\rho}(Z)=$ $\operatorname{dist}\left(Z, \Sigma_{\mathcal{D}, R / 2}\right)$ for any $Z \in \overline{\mathscr{C}}_{\Omega(z, R / 2)} \backslash \Sigma_{\mathcal{D}, R / 2}^{*}$.

Observe that if either (i) or (iii) holds true then the number $0<\delta(Z)$ in Theorem 3.5 has an infimum value, namely $0<\delta<\delta(Z)$ for any $Z \in \overline{\mathscr{C}}_{\Omega\left(z_{0}, R / 2\right)}$ and we deduce that solutions $W$ to problem (3.7) are Hölder continuous up to the boundary of $\mathscr{C}_{\Omega\left(z_{0}, R / 2\right)}$. In fact, let us consider two points $Z_{1}$ and $Z_{2}$ in $\overline{\mathscr{C}_{\Omega\left(z_{0}, R\right)}^{m}}$ with $m>0$. Then, by Corollary 3.1 and Theorem 3.5 we find

- If $\left|Z_{1}-Z_{2}\right| \geq \delta$, we have

$$
\frac{\left|W\left(Z_{1}\right)-W\left(Z_{2}\right)\right|}{\left|Z_{1}-Z_{2}\right|^{\tau}} \leq \frac{2}{\delta^{\tau}} \max _{\mathscr{C}_{\Omega\left(z_{0}, R / 2\right)}^{m}} W=\frac{2}{\delta^{\tau}}\|W\|_{L^{\infty}\left(\mathscr{C}_{\Omega\left(z_{0}, R / 2\right)}^{m}\right)} .
$$

- If $\left|Z_{1}-Z_{2}\right|<\delta$, by Theorem 3.5, $\frac{\left|W\left(Z_{1}\right)-W\left(Z_{2}\right)\right|}{\left|Z_{1}-Z_{2}\right|^{\tau}} \leq \mathcal{H}, 0<\mathcal{H}<1$.

We conclude the Hölder regularity with a constant

$$
\mathcal{T}=\max \left\{\mathcal{H}, \frac{2}{\delta^{\tau}}\|W\|_{L^{\infty}\left(\mathscr{C}_{\Omega(z, R / 2)}^{m}\right)}\right\} .
$$

Now we deal with the situation described in items (ii) and (iv).

Theorem 3.6. For any $z_{0} \in \Sigma_{\mathcal{D}}$ and $R>0$ let $W \in \mathcal{X}_{\Sigma_{\mathcal{D}, R}}^{s}\left(\mathscr{C}_{\Omega\left(z_{0}, R\right)}\right)$ be a solution to the homogeneous problem (3.7). Then $W \in \mathcal{C}_{\text {loc }}^{\tau}\left(\overline{\mathscr{C}}_{\Omega\left(z_{0}, R / 2\right)}\right)$ for some $0<\tau<\frac{1}{2}$.

Proof. Observe that the number $0<\delta(Z)$ in Theorem 3.5 is bounded from below by some $0<\delta_{\mathrm{H}}$ for $Z \in \Sigma_{\mathcal{D}, R / 2}^{*}$ and we can assume that $\delta(Z) \geq \min \left\{\delta_{\mathrm{H}}, \operatorname{dist}\left(Z, \Sigma_{\mathcal{D}, R / 2}^{*}\right)\right\}$ for $Z \in \Sigma_{\mathcal{N}, R / 2}^{*}$. Moreover, by the construction of the lateral boundary of the extension cylinder, the numbers $\delta(Z)$ do not depend on the $y$ variable. Hence such an infimum $\delta_{\mathrm{H}}>0$ is attained at those points of the type $Z=(z, 0)$ in $\partial \Omega \times\{0\}$. Consider the set

$$
\mathscr{C}_{\Omega\left(z_{0}, R / 2\right)}^{\delta}=\left\{Z \in \overline{\mathscr{C}_{\Omega(z, R / 2)}^{m}}: \operatorname{dist}\left(Z, \Sigma_{\mathcal{D}, R / 2}^{*}\right) \geq \delta_{\mathrm{H}}\right\} .
$$

As above, we only need to study the case $\left|Z_{1}-Z_{2}\right|<\delta_{\mathrm{H}}$. Suppose that $Z_{1} \in \mathscr{C}_{\Omega\left(z_{0}, R / 2\right)}^{\delta}$, then $\left|Z_{1}-Z_{2}\right| \leq \delta_{\mathrm{H}}<\operatorname{dist}\left(Z_{1}, \Sigma_{\mathcal{D}, R / 2}^{*}\right)=\delta\left(Z_{1}\right)$, and thus, by Theorem 3.5, we have

$$
\frac{\left|W\left(Z_{1}\right)-W\left(Z_{2}\right)\right|}{\left|Z_{1}-Z_{2}\right|^{\tau}} \leq \mathcal{H}
$$


If neither $Z_{1}$ nor $Z_{2}$ belongs to $\mathscr{C}_{\Omega\left(z_{0}, R / 2\right)}^{\delta}$ but one of them, say $Z_{1} \in \Sigma_{\mathcal{D}, R / 2}^{*}$, we have $\left|Z_{1}-Z_{2}\right| \leq$ $\delta_{\mathrm{H}}=\delta\left(Z_{1}\right)$, and the results follows as before. If, instead, none of them belongs neither to $\mathscr{C}_{\Omega\left(z_{0}, R / 2\right)}^{\delta}$ nor to $\Sigma_{\mathcal{D}, R / 2}^{*}$, we have two cases:

$$
\begin{aligned}
& \text { - }\left|Z_{1}-Z_{2}\right| \leq \max \left\{\operatorname{dist}\left(Z_{1}, \Sigma_{\mathcal{D}, R / 2}^{*}\right), \operatorname{dist}\left(Z_{2}, \Sigma_{\mathcal{D}, R / 2}^{*}\right)\right\} . \\
& \text { - }\left|Z_{1}-Z_{2}\right|>\max \left\{\operatorname{dist}\left(Z_{1}, \Sigma_{\mathcal{D}, R / 2}^{*}\right), \operatorname{dist}\left(Z_{2}, \Sigma_{\mathcal{D}, R / 2}^{*}\right)\right\} .
\end{aligned}
$$

In the first case at least one of the two points, say $Z_{1}$, satisfies the inequality $\left|Z_{1}-Z_{2}\right| \leq \delta_{\mathrm{H}}<$ $\operatorname{dist}\left(Z_{1}, \Sigma_{\mathcal{D}, R / 2}^{*}\right)=\delta\left(Z_{1}\right)$ and we have the result as before. In the second case, there exists at least one $\bar{Z} \in \Sigma_{\mathcal{D}, R / 2}^{*}$ such that $\left|\bar{Z}-Z_{1}\right| \leq\left|Z_{1}-Z_{2}\right|$, and using the triangle inequality it follows that $\left|\bar{Z}-Z_{2}\right| \leq 2\left|Z_{1}-Z_{2}\right|$. Since the result has been proved for the case when at least one point belongs to $\Sigma_{\mathcal{D}, R / 2}^{*}$, we find

$$
\left|W\left(Z_{1}\right)-W\left(Z_{2}\right)\right| \leq\left|W\left(Z_{1}\right)-W(\bar{Z})\right|+\left|W(\bar{Z})-W\left(Z_{2}\right)\right| \leq 3 \mathcal{H}\left|Z_{1}-Z_{2}\right|^{\tau},
$$

and we conclude the Hölder regularity with constant $\mathcal{T}=\max \left\{3 \mathcal{H}, 2 \delta_{\mathrm{H}}^{-\tau}\|W\|_{L^{\infty}\left(\mathscr{C}_{\Omega(z, R / 2)}\right)}\right\}$, with $0<\mathcal{H}<1$ given by Theorem 3.5 , see (3.34)

Corollary 3.2. Let $\Omega$ be a smooth domain such that $\Sigma_{\mathcal{D}}, \Sigma_{\mathcal{N}}$ satisfy hypotheses ( $\left.\mathfrak{B}\right)$ and let $w$ be the solution to problem (3.5) with $z \in \bar{\Omega}$ and $R>0$. Then, the function $w \in \mathcal{C}^{\tau}(\overline{\Omega(z, R / 2)})$ for some $0<\tau<\frac{1}{2}$.

Proof. Since $\Omega$ satisfies hypotheses $(\mathfrak{B})$, there exists $0<\delta_{\mathrm{H}}<\delta(Z)$ for $Z \in \Sigma_{\mathcal{D}, R / 2}^{*}$ and we can assume that $\delta(Z) \geq \min \left\{\delta_{\mathrm{H}}, \operatorname{dist}\left(Z, \Sigma_{\mathcal{D}, R / 2}^{*}\right)\right\}$ for $Z \in \Sigma_{\mathcal{N}, R / 2}^{*}$, with $\delta(Z)$ given in Theorem 3.5 Suppose that $z_{1}, z_{2} \in(\overline{\Omega(z, R / 2)})$ :

- If $\left|z_{1}-z_{2}\right| \geq \delta_{\mathrm{H}}$. Then, due to Corollary 3.1 we have $\|w\|_{L^{\infty}(\Omega(z, R / 2))}<\infty$ and, therefore,

$$
\frac{\left|w\left(z_{1}\right)-w\left(z_{2}\right)\right|}{\left|z_{1}-z_{2}\right|^{\tau}} \leq \frac{2}{\delta_{\mathrm{H}}^{\tau}} \max _{\Omega(z, R / 2)} w .
$$

- While for $\left|z_{1}-z_{2}\right|<\delta_{\mathrm{H}}$, let us set $Z_{1}=\left(z_{1}, 0\right)$ and $Z_{2}=\left(z_{2}, 0\right), Z_{1}, Z_{2} \in \overline{\mathscr{C}}_{\Omega(z, R / 2)}$, such that $\left|Z_{1}-Z_{2}\right|<\delta_{\mathrm{H}}$. Then, as in 3.35) in Theorem 3.6.

$$
\frac{\left|w\left(z_{1}\right)-w\left(z_{2}\right)\right|}{\left|z_{1}-z_{2}\right|^{\tau}}=\frac{\left|W\left(Z_{1}\right)-W\left(Z_{2}\right)\right|}{\left|Z_{1}-Z_{2}\right|^{\tau}} \leq 3 \mathcal{H}, \quad 0<\mathcal{H}<1 .
$$

Hence, we conclude

$$
\left|w\left(z_{1}\right)-w\left(z_{2}\right)\right| \leq \mathcal{T}\left|z_{1}-z_{2}\right|^{\tau}, \quad \forall z_{1}, z_{2} \in \overline{\Omega(z, R / 2)}
$$

with $\mathcal{T}=\max \left\{3 \mathcal{H}, 2 \delta_{\mathrm{H}}^{-\tau}\|w\|_{L^{\infty}(\Omega(z, R / 2))}\right\}$, and $\delta_{\mathrm{H}}>0$ given as above.

We prove now the main result of this work.

Proof of Theorem 1.1. Let $u$ be the solution to problem $\left(\overline{P^{s}}\right), \Omega$ a smooth bounded domain such that $\Sigma_{\mathcal{D}}, \Sigma_{\mathcal{N}}$ satisfy hypotheses $(\mathfrak{B})$ and $f \in L^{p}(\Omega)$ for $p>\frac{N}{2 s}$. Given $z \in \bar{\Omega}$ and $0<R<1$, let $v$ be the solution to (3.4) and $w=u-v$ a function satisfying (3.5). Thus, using (3.13) and Corollary 3.2 . we conclude that, for any $x, y \in \overline{\Omega(z, R / 2)}$,

$$
\omega(u, R / 2) \leq \omega(w, R / 2)+2 \max _{x \in \Omega(z, R / 2)} v(x) \leq \mathcal{T} R^{\tau}+C\left(N, s,\left|\Sigma_{\mathcal{D}}\right|\right)\|f\|_{L^{p}(\Omega(z, R))} R^{2 s-\frac{N}{p}} \leq \mathcal{C} R^{\gamma},
$$

where $\gamma=\min \left\{\tau, 2 s-\frac{N}{p}\right\}<\frac{1}{2}$ and $\mathcal{C}=\max \left\{\mathcal{T}, 2 C\left(N, s,\left|\Sigma_{\mathcal{D}}\right|\right)\|f\|_{L^{p}(\Omega(z, R))}\right\}$, with

$$
\mathcal{T}=\max \left\{3 \mathcal{H}, 2 \delta_{\mathrm{H}}^{-\tau}\|w\|_{L^{\infty}(\Omega(z, R / 2))}\right\}=\max \left\{3 \mathcal{H}, 2 \delta_{\mathrm{H}}^{-\tau}\|u-v\|_{L^{\infty}(\Omega(z, R / 2))}\right\} .
$$

Moreover, by Theorem[3.2. $\|u-v\|_{L^{\infty}(\Omega(z, R / 2))} \leq\|u\|_{L^{\infty}(\Omega(z, R))}+\|v\|_{L^{\infty}(\Omega(z, R))} \leq 2 C\left(N, s,\left|\Sigma_{\mathcal{D}}\right|\right)\|f\|_{L^{p}(\Omega(z, R))}$ hence we obtain

$$
\mathcal{T} \leq \max \left\{3 \mathcal{H}, 4 \delta_{\mathrm{H}}^{-\tau} C\left(N, s,\left|\Sigma_{\mathcal{D}}\right|\right)\|f\|_{p}\right\}
$$


Therefore, $\mathcal{C}=\max \left\{3 \mathcal{H}, 4 \delta_{\mathrm{H}}^{-\tau} C\left(N, s,\left|\Sigma_{\mathcal{D}}\right|\right)\|f\|_{L^{p}(\Omega(z, R))}\right\}$. Repeating the steps above in Theorem [3.6, we conclude

$$
|u(x)-u(y)| \leq \mathscr{H}|x-y|^{\gamma}, \text { for any } x, y \in \overline{\Omega(z, R / 2)},
$$

where

$$
\mathscr{H}=\max \left\{9 \mathcal{H}, \frac{C\left(N, s,\left|\Sigma_{\mathcal{D}}\right|\right)\|f\|_{L^{p}(\Omega(z, R))}}{\delta_{\mathrm{H}}^{\gamma}}\right\},
$$

and $\gamma=\min \left\{\tau, 2 s-\frac{N}{p}\right\}<\frac{1}{2}$. Since the constants $\mathscr{H}$ and $\gamma$ do not depend neither on $z$ nor on $R$, to complete the proof, set $z_{i} \in \bar{\Omega}, i=1,2, \ldots, m$ and $R_{i}>0$, small enough such that

$$
\bar{\Omega}=\bigcup_{i=1}^{m} \Omega\left(z_{i}, R_{i} / 4\right)
$$

Then (3.36) follows by using a suitable recovering argument.

\section{Moving the Boundary COnditions}

In this last part, we study the behavior of the solutions to problem $\left(P^{s}\right)$ when we move the boundary conditions. First, let us describe this mixed moving boundary data framework. As introduced above, given $I_{\varepsilon}=[\varepsilon,|\partial \Omega|]$, let us consider the family of closed sets $\left\{\Sigma_{\mathcal{D}}(\alpha)\right\}_{\alpha \in I_{\varepsilon}}$, satisfying

$\left(B_{1}\right) \Sigma_{\mathcal{D}}(\alpha)$ has a finite number of connected components.

$\left(B_{2}\right) \Sigma_{\mathcal{D}}\left(\alpha_{1}\right) \subset \Sigma_{\mathcal{D}}\left(\alpha_{2}\right)$ if $\alpha_{1}<\alpha_{2}$.

$\left(B_{3}\right)\left|\Sigma_{\mathcal{D}}\left(\alpha_{1}\right)\right|=\alpha_{1} \in I_{\varepsilon}$.

We call $\Sigma_{\mathcal{N}}(\alpha)=\partial \Omega \backslash \Sigma_{\mathcal{D}}(\alpha)$ and $\Gamma(\alpha)=\Sigma_{\mathcal{D}}(\alpha) \cap \bar{\Sigma}_{\mathcal{N}}(\alpha)$. Observe that, under the hypotheses $\left(B_{1}\right)-$ $\left(B_{3}\right)$, the limit sets $\Sigma_{\mathcal{D}}(\alpha), \Sigma_{\mathcal{N}}(\alpha)$ as $\alpha \rightarrow \varepsilon^{+}$are not degenerated sets (for instance a Cantor-like set).

For a family of this type we consider the corresponding family of mixed boundary value problems

$$
\left\{\begin{aligned}
(-\Delta)^{s} u=f & \text { in } \Omega, \\
B_{\alpha}(u)=0 & \text { on } \partial \Omega,
\end{aligned}\right.
$$

where $B_{\alpha}(u)$ means $B(u)$ with $\Sigma_{\mathcal{D}}, \Sigma_{\mathcal{N}}$, and $\Gamma$ are replaced by $\Sigma_{\mathcal{D}}(\alpha), \Sigma_{\mathcal{N}}(\alpha)$, and $\Gamma(\alpha)$ respectively. Similarly, $\left(\mathfrak{B}_{\alpha}\right)$ means $(\mathfrak{B})$ with the natural changes as above.

Our main aim here is to prove Theorem 1.2

The key point in order to obtain it, is to prove that we can choose $\beta_{s}>0$ in (3.11) independent of the measure of the Dirichlet part. Nevertheless, as we will see below, when one takes $\alpha \rightarrow 0^{+}$the control of the Hölder norm of such a family is lost. Hence, it is necessary to fix a positive minimum $\varepsilon>0$ on the measure of the family $\left\{\Sigma_{\mathcal{D}}(\alpha)\right\}_{\alpha \in I_{\varepsilon}}$, in order to guarantee the control on the Hölder norm for the family $\left\{u_{\alpha}\right\}_{\alpha \in I_{\varepsilon}}$.

Proof of Theorem 1.2. Assume that $\partial \Omega$ is a smooth manifold and $\Sigma_{\mathcal{D}}(\alpha), \Sigma_{\mathcal{N}}(\alpha)$ satisfy hypotheses $(\mathfrak{B})$. Thus, there exists $\delta>0$ such that $\bar{\rho}(Z) \geq \delta$ for all $Z \in \partial_{L} \mathscr{C}_{\Omega}$. Then:

(1) If $Z \in \overline{\mathscr{C}}_{\Omega} \backslash \Sigma_{\mathcal{D}}^{*}(\alpha)$, inequality (3.11) holds true with $\beta_{s}=\frac{c_{s}}{\zeta \lambda}$ independent of $\alpha$, for all $0<\rho<\delta$.

(2) If $Z \in \Sigma_{\mathcal{D}}^{*}(\alpha) \backslash \Gamma^{*}(\alpha)$, we can set $0<\rho<\min \left\{\delta\right.$, $\left.\operatorname{dist}\left(Z, \Gamma^{*}(\alpha)\right)\right\}$, such that for all $X \in$ $\mathscr{C}_{\Omega}(Z, \rho)$

$$
\Pi\left(X, \Sigma_{\mathcal{D}}^{*} \cap B_{\rho}(Z), \mathscr{C}_{\Omega}(Z, \rho)\right) \geq \varphi>0,
$$

with $\varphi$ independent from $\alpha$, recalling that (according to [10, §4])

$$
\Pi\left(x_{0}, E, A\right)=\left|\mathcal{V}_{x_{0}}(E) \cap \mathbb{S}_{N-1}\left(x_{0}\right)\right|=\left|\mathscr{S}_{x_{0}}\right| .
$$

with $\mathcal{V}$ defined as follows: given $x_{0} \in A$ and a closed set $E \subset A$, let us consider the cone $\mathcal{V}_{x_{0}}(E) \subset A$ consisting on all rays starting at $x_{0}$ and ending at some point $P \in E$.

Hence, inequality (3.11) holds true with $\beta_{s} \leq \frac{c_{s}}{\varphi}$ also independent from $\alpha$. 
(3) If $Z \in \Gamma^{*}(\alpha)$, we can assume without loss of generality that, for some neighborhood of radius $0<\rho<\min \left\{\delta, \delta_{\Gamma}\right\}$ of the point $Z=\left(Z_{1}, \ldots, Z_{N+1}\right), \partial_{L} \mathscr{C}_{\Omega}$ coincides with the hyperplane $\mathbb{R}^{N+1} \cap\left\{x_{N}=0\right\}$ and $\Gamma^{*}(\alpha) \subset \mathbb{R}_{+}^{N+1} \cap\left\{x_{N}=0, x_{N-1}=0\right\}$, in such a way that in $\Sigma_{\mathcal{D}}^{*}(\alpha)$ we have $x_{N-1} \geq 0$ and, in $\Sigma_{\mathcal{N}}^{*}(\alpha)$ we have $x_{N-1}<0$. Now, $\mathscr{C}_{\Omega}(Z, \rho)$ is transformed by the bi-Lipschitz transform (that in fact keeps the extension variable unchanged)

$$
\begin{gathered}
x_{i}=\xi_{i}, \quad i=1,2, \ldots, N-1, \\
x_{N}= \begin{cases}\xi_{N} & \text { if } \xi_{N-1}<0, \\
\xi_{N}-\xi_{N-1} & \text { if } \xi_{N-1} \geq 0,\end{cases}
\end{gathered}
$$

into a set $\mathcal{O}_{\rho}(Z)=\mathcal{O}_{\rho}^{1}(Z) \cup \mathcal{O}_{\rho}^{2}(Z)$ with

$$
\begin{aligned}
& \mathcal{O}_{\rho}^{1}(Z)=\left\{\xi_{N} \geq 0, \xi_{N-1}<0, \sum_{i=1}^{N}\left(\xi_{i}-Z_{i}\right)^{2}+\left(y-Z_{N+1}\right)^{2} \leq \rho^{2}\right\}, \\
& \mathcal{O}_{\rho}^{2}(Z)=\left\{\begin{array}{l}
\xi_{N-1} \geq 0, \sum_{i=1}^{N-1}\left(\xi_{i}-Z_{i}\right)^{2}+\left(y-Z_{N+1}\right)^{2} \leq \rho^{2}, \\
\xi_{N-1} \leq \xi_{N} \leq \xi_{N-1}+\left(\rho^{2}-\sum_{i=1}^{N-1}\left(\xi_{i}-Z_{i}\right)^{2}-\left(y-Z_{N+1}\right)^{2}\right)^{\frac{1}{2}}
\end{array}\right\} .
\end{aligned}
$$

Moreover $\Sigma_{\mathcal{D}}^{*} \cap B_{\rho}(Z)$ is transformed into the set

$$
\mathcal{D}_{\rho}(Z)=\left\{\xi_{N}=\xi_{N-1}, \xi_{N-1} \geq 0, \sum_{i=1}^{N-1}\left(\xi_{i}-Z_{i}\right)^{2}+\left(y-Z_{N+1}\right)^{2} \leq \rho^{2}\right\} .
$$

Given $X_{0} \in \mathcal{O}_{\rho}(Z)$, we use again the representation (see [10, cfr. 13.1]):

$$
\Pi\left(X_{0}, \mathcal{D}_{\rho}(Z), \mathcal{O}_{\rho}(Z)\right)=\frac{1}{\left|\mathbb{S}_{N}\left(X_{0}\right)\right|} \int_{\mathcal{D}_{\rho}(Z)} \frac{1}{\left|X_{0}-Y\right|^{N}} \cos (\psi) d \sigma
$$

where $\cos (\psi)=\left\langle\frac{X_{0}-Y}{\left|X_{0}-Y\right|}, \vec{v}\right\rangle$, with $\vec{v}$ the normal vector to $\left\{\xi_{N}=\xi_{N-1}\right\} \cap \mathbb{R}_{+}^{N+1}$. Since $\cos (\psi)$ vanish only when $X_{0} \in \mathcal{D}_{\rho}(Z)$ we conclude that $\Pi\left(X_{0}, \mathcal{D}_{\rho}(Z), \mathbb{R}_{+}^{N+1}\right) \geq \varphi>0$ for all $X_{0} \in \mathcal{O}_{\rho}(Z)$ and some $\varphi>0$ independent of $\alpha$. On the other hand, it is immediate that $\varphi$ is independent of $\rho$. Hence, inequality (3.11) holds true with $\beta_{s} \leq \frac{c_{s}}{\varphi}$ also independent of $\alpha$.

Let us define

$$
\bar{\rho}_{\alpha}(Z):= \begin{cases}\min \left\{\delta, \operatorname{dist}\left(Z, \Sigma_{\mathcal{D}}^{*}\right)\right\}, & \text { if } Z \in \overline{\mathscr{C}}_{\Omega} \backslash \Sigma_{\mathcal{D}}^{*}(\alpha), \\ \min \left\{\delta, \operatorname{dist}\left(Z, \Gamma^{*}\right)\right\}, & \text { if } Z \in \Sigma_{\mathcal{D}}^{*}(\alpha) \backslash \Gamma^{*}(\alpha), \\ \min \left\{\delta, \delta_{\Gamma}\right\}, & \text { if } Z \in \Gamma^{*}(\alpha) .\end{cases}
$$

As a consequence of (1)-(3) above, we deduce

(i) by (3.26), the constant $\Lambda$ appearing in Theorem 3.3 and Theorem 3.4 is independent of $\alpha$. Hence, inequality (3.28) does not depends on $\alpha$ and also the number $0<\mathcal{H}<1$ in Theorem 3.5 is independent from $\alpha$.

(ii) by (3.32), the constant $\bar{\eta}$ in Theorem 3.4 is independent from $\alpha$ and, by (3.33), also that $0<\gamma<\frac{1}{2}$ is independent from $\alpha$.

Then, given $u_{\alpha}$ a solution to problem $\left[P_{\alpha}^{s}\right.$ with $\alpha \in I_{\varepsilon}$, by Theorem 1.1] we deduce

$$
\left\|u_{\alpha}\right\|_{\mathcal{C}^{\gamma}(\Omega)} \leq \mathscr{H}_{\alpha}
$$

with $\gamma=\min \left\{\tau, 2 s-\frac{N}{p}\right\}<\frac{1}{2}$ independent of $\alpha$ and $\mathscr{H}_{\alpha}=\max \left\{9 \mathcal{H}, \frac{C(N, s, \alpha)\|f\|_{p}}{\delta_{\mathrm{H}, \alpha}^{\tau}}\right\}$ with the constants $0<\tau<\frac{1}{2}$ and $\delta_{\mathrm{H}, \alpha}$ given as in Corollary 3.2. Now, if we consider the family $\left\{u_{\alpha}\right\}_{\alpha \in I_{\varepsilon}}$, since $\bar{\rho}_{\alpha_{1}}(Z) \leq \bar{\rho}_{\alpha_{2}}(Z)$ it is clear that $\delta_{\mathrm{H}, \alpha_{1}} \leq \delta_{\mathrm{H}, \alpha_{2}}$ and, therefore, $\mathscr{H}_{\alpha_{1}} \geq \mathscr{H}_{\alpha_{2}}$ for all $\alpha_{1}, \alpha_{2} \in[\varepsilon,|\partial \Omega|]$, $\alpha_{1} \leq \alpha_{2}$. Therefore, we can take $0<\gamma<\frac{1}{2}$ and $\mathscr{H}_{\varepsilon}=\max \left\{9 \mathcal{H}, \frac{C(N, s, \varepsilon)\|f\|_{p}}{\delta_{\mathrm{H}, \varepsilon}^{r}}\right\}$ independent from $\alpha$ such that

$$
\left\|u_{\alpha}\right\|_{\mathcal{C}^{\gamma}(\Omega)} \leq \mathscr{H}_{\varepsilon}
$$


To conclude, we observe that the condition $\alpha \in[\varepsilon,|\partial \Omega|]$ is necessary in order to control the Hölder norm of the family $\left\{u_{\alpha}\right\}_{\alpha \in I_{\varepsilon}}$. If we let $\alpha=\left|\Sigma_{\mathcal{D}}(\alpha)\right| \rightarrow 0^{+}$, then it is clear that $\left|\Sigma_{\mathcal{D}}^{*}(\alpha) \cap \overline{\mathscr{C}}_{\Omega}(Z, \rho)\right| \rightarrow 0$ for any $Z \in \overline{\mathscr{C}}_{\Omega}$ and $\rho>0$. Thus, if $\alpha \rightarrow 0^{+}$, we conclude from (4.1) that $\bar{\rho}_{\alpha}(Z) \rightarrow 0$ for any $Z \in \Sigma_{\mathcal{D}}^{*}$ and, hence, $\delta_{\mathrm{H}, \alpha} \rightarrow 0$ while $\mathscr{H}_{\alpha} \rightarrow+\infty$.

Remark 4.1. Given an interphase point $Z \in \Gamma^{*}$, it is clear from (4.1), that we can choose an uniform $\rho_{\varepsilon}>0$ in the lines of [6, Corollary 6.1]. In fact, it is enough to choose $\delta_{\Gamma}$ in (4.1) in such a way that $\Sigma_{\mathcal{D}}^{*}(\varepsilon) \cap \overline{\mathscr{C}}_{\Omega}(Z, \rho)$ is contained in some hyperplane (see (3) in the proof of Theorem 1.2). Clearly, this Dirichlet boundary part, say $\left(\left\{x_{N}=0, x_{N-1} \geq 0\right\} \cap \mathbb{R}_{+}^{N+1}\right) \cap B_{\rho_{\varepsilon}}(Z)$ converges to an empty set as $\rho_{\varepsilon} \rightarrow 0$.

\section{REFERENCES}

[1] C. Brandle. E. Colorado, A. de Pablo, U. Sanchez, A concave-convex elliptic problem involving the fractional Laplacian, Proc. Roy. Soc. Edinburgh Sect. A 143 (2013), 39?71

[2] L. Caffarelli, L.Silvestre, An extension problem related to the fractional Laplacian, Comm. Partial Differential Equations, 32 (2007), 1245-1260.

[3] X. Cabré, J. Tan, Positive solutions of nonlinear problems involving the square root of the Laplacian. Adv. Math, 224 (2010), 2052-2093.

[4] J. Carmona, E. Colorado, T. Leonori, A. Ortega, Semilinear fractional elliptic problems with mixed DirichletNeumann boundary conditions. Preprint, arXiv:1902.08925v1.

[5] E. Colorado, A. Ortega, The Brezis-Nirenberg problem for the fractional Laplacian with mixed Dirichlet-Neumann boundary conditions, J. Math. Anal. Appl. 473 (2019), 1002-1025.

[6] E. Colorado, I. Peral, Semilinear elliptic problems with mixed Dirichlet-Neumann boundary conditions, Journal of Functional Analysis, 199 (2003), 468-507.

[7] E. B. Fabes, C. E. Kenig, R. P. Serapioni, The local regularity of solutions of degenerate elliptic equations, Comm. in Partial Differential Equations, 7(1982), 77-116.

[8] D. Kinderlehrer, G. Stampacchia, An introduction to Variational Inequalities an Their Applications, (Pure and Applied Mathematics 88) (Eds.)-Academic Press, Elsevier (1980).

[9] J.-L. Lions, E. Magenes, Non-homogeneous boundary value problems and applications., Vol. I , (Translated from the French by P. Kenneth) GMW 181, Springer-Verlag, New York-Heidelberg, 1972. xvi+357 pp.

[10] G. Stampacchia, Problemi al contorno ellittici, con dati discontinui, dotati di soluzioni Holderiane, Ann. Mat. Pura Appl. 51 (1960) 1-37.

[11] E. M. Stein, Harmonic Analysis: Real-Variable Methods, Orthogonality, and Oscillatory Integrals, Princeton University Press (1993).

(J. Carmona) Departamento de Matemáticas, Universidad de Almería,

Ctra. Sacramento s/n, La Cañada de San Urbano, 04120 Almería, Spain

E-mail address: jcarmona@ual.es

(E. Colorado, A. Ortega) Departamento de Matemáticas, Universidad Carlos iII de Madrid,

Av. Universidad 30, 28911 Leganés (Madrid), Spain

E-mail address, E. Colorado: ecolorad@math.uc3m.es

E-mail address, A. Ortega: alortega@math.uc3m.es

(T. Leonori) Dipartimento di Scienze di Base e Applicate per l'Ingegneria Università di Roma "Sapienza" Via Antonio Scarpa 10, 00161 Roma

E-mail address: tommaso.leonori@sbai.uniroma1.it 